\title{
Optimum tuning of series and parallel LR circuits for passive vibration suppression using piezoelectric elements
}

\section{$\operatorname{AUTHOR}(\mathrm{S}):$}

Yamada, Keisuke; Matsuhisa, Hiroshi; Utsuno, Hideo; Sawada, Katsutoshi

\section{CITATION:}

Yamada, Keisuke ... [et al]. Optimum tuning of series and parallel LR circuits for passive vibration suppression using piezoelectric elements. Journal of Sound and Vibration 2010, 329(24): 5036-5057

ISSUE DATE:

2010-11-22

URL:

http://hdl.handle.net/2433/128768

\section{RIGHT:}

(C) 2010 Elsevier Ltd; この論文は出版社版でありません。引用の際には 出版社版をご確認ご利用ください。; This is not the published version. Please cite only the published version. 


\title{
Optimum tuning of series and parallel LR circuits for passive vibration suppression using piezoelectric elements
}

\author{
Keisuke YAMADA*, Hiroshi MATSUHISA, Hideo UTSUNO, Katsutoshi SAWADA \\ *Department of Mechanical Engineering and Science, Kyoto University, Yoshida-Honmachi, Sakyo-ku, Kyoto \\ 606-8501, Japan
}

\begin{abstract}
Bending vibration of flexible structures can be suppressed passively using piezoelectric electromechanical transducers and optimally tuned LR circuits. Since these systems include both mechanical and electrical elements, the governing equations consist of electrically coupled equations of motion. This paper describes a new method for deriving the governing equations that describe a system's vibration suppression based on the equilibrium of force principle and using an equivalent mechanical model of a piezoelectric element. Both series and parallel LR circuits are considered in the modeling approach. The optimum values for a mechanical vibration absorber can be formulated by using the two fixed points method. However, exact optimal values for the resistances of the LR circuits have not been formulated in the research literature thus far, and approximate values have been used. Analytical formulations are derived in this paper, and optimum values of the LR circuits are presented, not only in displacement, but also in terms of velocity and acceleration. The effects of the stiffness of the adhesive bond between the host structure and piezoelectric element, the dielectric loss in a piezoelectric element, and the internal resistance of an inductor are considered in the theoretical analysis. The effectiveness of the described analytical method is validated through simulations and experiments.
\end{abstract}

Key Words: Vibration Control, Piezoelectric Element, Dynamic Absorber, Flexible Structure, Smart Structure

\section{Introduction}

Suppression of bending vibration using piezoelectric elements has attracted the attention of many researchers. Typically, thin ceramic plates of piezoelectric material are used because this configuration requires minimal additional space and they are easy to install. In contrast, vibration suppression devices such as mechanical vibration absorbers usually need a significant amount of space and require a system level integration approach. Both active vibration control [1,2] and passive vibration suppression [3-7] using piezoelectric elements have been investigated in the research literature. Several hybrid methods [8-11] have also been proposed. Active 
vibration control is often more effective than passive vibration suppression for a given device size, but they suffer from stability problems. In principle, passive methods are stable and offer a higher degree of simplicity in their implementation. For these reasons, passive vibration suppression using tuned LR circuits is the focus of this paper.

In previous works, it has been shown that both series and parallel LR circuits are effective in passively absorbing vibration using piezoelectric elements $[4,6]$. Fundamental characterization of piezoelectric vibration absorbers and passive LR circuits has been shown; however, significant work remains in specific areas. In particular, the characteristics of the piezoelectric elements have not been thoroughly included in the derivation of governing equations, and as a result, the mechanism for how the controlling force is generated by LR circuits has not been illustrated in detail. In addition, accurate formulations for obtaining optimum values for the resistances, which agree with the two fixed points method [12], have not been derived thus far. The differences in performance between series and parallel LR circuits should be investigated more closely. In the research literature, the optimum LR values for the circuits were derived only with respect to displacement even though the vibration of the host structure is often evaluated in terms of velocity or acceleration. Finally, the results of pure theoretical analysis and experiment often do not agree well in the research literature, especially in terms of the equivalent stiffness ratio of the piezoelectric element and the optimum value of resistance. To address these issues, this paper derives the governing equations by using a new equivalent mechanical model of a piezoelectric element. An equivalent model of a piezoelectric element proposed previously consists of frequency-dependent elements [13]; however, the proposed equivalent model consists of frequency-independent elements. Using the two fixed points method, accurate formulations for the optimum values of the LR circuits are derived, not only in terms of displacement but also in terms of velocity and acceleration. Using these formulations, the performance and optimum values of series and parallel LR circuits are compared theoretically. Finally, the dielectric loss of a piezoelectric element, internal resistance of the inductor, and stiffness of the adhesive bond are modeled theoretically. The effectiveness of the theoretical analysis is verified in simulations and experiments.

\section{Theoretical analysis}

\subsection{Piezoelectric constitutive equations and equivalent mechanical model}

Piezoelectric elements generate electrical voltage when they are strained. This phenomenon is called the piezoelectric effect. The inverse piezoelectric effect occurs when a piezoelectric element strains in response to an applied voltage. A piezoelectric element can be used as both a sensor and an actuator by using these responses. Piezoelectric elements can be categorized into several types according to the directions of polarization and strain. There is no essential difference between them and general formulations will be developed throughout this work. A plate type of piezoelectric elements used in this paper is usually used for bending vibration suppression. As shown in Fig. 1, directions of polarization and strain of the plate type are perpendicular to each other. The piezoelectric constitutive equations are given as 


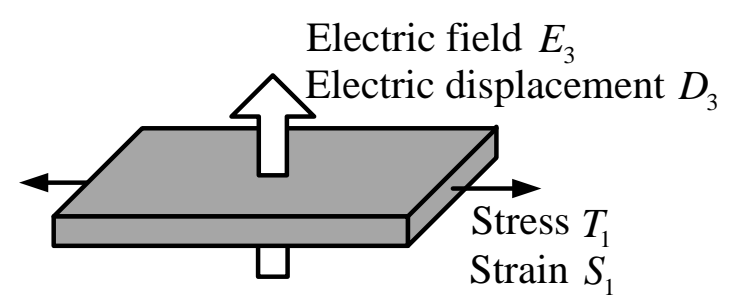

Fig. 1. Schematic diagram of a plate type piezoelectric element.

$$
\begin{aligned}
& S_{1}=s_{11}^{E} T_{1}+d_{31} E_{3}, \\
& D_{3}=d_{31} T_{1}+\varepsilon_{33}^{T} E_{3},
\end{aligned}
$$

where $S_{1}$ is the strain, $T_{1}$ is the stress, $E_{3}$ is the electrical field, $D_{3}$ is the electrical displacement, $s_{11}^{E}$ is the elastic compliance defined as the reciprocal of Young's modulus, $d_{31}$ is the piezoelectric constant, and $\varepsilon_{33}^{T}$ is the electrical permittivity. The subscripts 1 and 11 denote the longitudinal direction, 3 and 33 the thickness direction, and 31 that the electrical displacement is the thickness direction and the strain is the longitudinal direction. The superscripts $E$ and $T$ denote the values which are obtained under constant electrical field and constant stress, respectively. The strain in width direction and the mass of the piezoelectric element are ignored here for simplicity. Equations (1) and (2) describe the inverse piezoelectric effect and piezoelectric effect, respectively. Equations (1) and (2) can be transformed into the following equations:

$$
\begin{gathered}
x_{1}=\frac{1}{E_{\mathrm{p}}} \frac{l_{\mathrm{p}}}{w_{\mathrm{p}} t_{\mathrm{p}}} F_{1}+d_{31} \frac{l_{\mathrm{p}}}{t_{\mathrm{p}}} V_{3}, \\
q_{3}=d_{31} \frac{l_{\mathrm{p}}}{t_{\mathrm{p}}} F_{1}+\varepsilon_{33}^{T} \frac{l_{\mathrm{p}} w_{\mathrm{p}}}{t_{\mathrm{p}}} V_{3}, \\
x_{1}=l_{\mathrm{p}} S_{1}, \quad q_{3}=l_{\mathrm{p}} w_{\mathrm{p}} D_{3}, \quad F_{1}=w_{\mathrm{p}} t_{\mathrm{p}} T_{1}, \quad V_{3}=t_{\mathrm{p}} E_{3},
\end{gathered}
$$

where $F_{1}$ is the force, $V_{3}$ is the voltage, $x_{1}$ is the displacement, $q_{3}$ is the charge, $E_{\mathrm{p}}$ is the Young's modulus defined as reciprocal of $s_{11}^{E}, l_{\mathrm{p}}, w_{\mathrm{p}}$, and $t_{\mathrm{p}}$ are the length, width, and thickness, respectively. Equations (3) and (4) are simplified as

$$
\begin{gathered}
x_{1}=\frac{1}{k_{\mathrm{p}}} F_{1}+\frac{\theta_{\mathrm{p}}}{k_{\mathrm{p}}} V_{3}, \\
q_{3}=\frac{\theta_{\mathrm{p}}}{k_{\mathrm{p}}} F_{1}+C_{\mathrm{p}}^{T} V_{3}, \\
k_{\mathrm{p}}=E_{\mathrm{p}} \frac{w_{\mathrm{p}} t_{\mathrm{p}}}{l_{\mathrm{p}}}, \theta_{\mathrm{p}}=d_{31} E_{\mathrm{p}} w_{\mathrm{p}}, C_{\mathrm{p}}^{T}=\varepsilon_{33}^{T} \frac{l_{\mathrm{p}} w_{\mathrm{p}}}{t_{\mathrm{p}}},
\end{gathered}
$$

where $k_{\mathrm{p}}$ is the longitudinal mechanical stiffness, $\theta_{\mathrm{p}}$ is the elemental electromechanical coupling coefficient, and $C_{\mathrm{p}}^{T}$ is the capacitance under constant stress. From Eqs. (9) and (10), capacitance under constant strain is defined as follows.

$$
C_{\mathrm{p}}^{S}=C_{\mathrm{p}}^{T}-\frac{\theta_{\mathrm{p}}^{2}}{k_{\mathrm{p}}},
$$

where the superscript $S$ denotes that the value is obtained under constant strain. Substituting Eq. (14) into Eq. 
(10) gives

$$
q_{3}=\frac{\theta_{\mathrm{p}}}{k_{\mathrm{p}}} F_{1}+\left(\frac{\theta_{\mathrm{p}}^{2}}{k_{\mathrm{p}}}+C_{\mathrm{p}}^{S}\right) V_{3}=\theta_{\mathrm{p}} x_{1}+C_{\mathrm{p}}^{S} V_{3} .
$$

From Eqs. (9) and (15), the equivalent mechanical model is drawn as Fig. 2 (a). Here $1 / \bar{C}_{\mathrm{p}}^{s}$ is the spring constant, $\bar{\theta}_{\mathrm{p}}$ is the area ratio between the upper and right pistons, $\bar{V}_{3}$ is the force, $\bar{q}_{3}$ is the displacement. The values of $\bar{C}_{\mathrm{p}}^{S}, \bar{\theta}_{\mathrm{p}}, \bar{V}_{3}$, and $\bar{q}_{3}$ are equal to the values of $C_{\mathrm{p}}^{S}, \theta_{\mathrm{p}}, V_{3}$, and $q_{3}$, respectively. The left half of the equivalent model (a) is the mechanical stiffness given by Eq. (11), and the right half shows the electrical properties of the transducer used to convert between mechanical and electrical energy. The volume in the cylinder is constant, and the pressure in the cylinder is uniform. The cylinder is fixed, and does not move. The equivalent mechanical model (a) can be transformed into the mechanical model (b). In this paper, the equivalent mechanical models (a) and (b) shown in Fig. 2 are referred to subsequently as imaginary equivalent mechanical model and the perfect equivalent mechanical model, respectively. The stiffness of the electrical part is written as

$$
k_{\mathrm{z}}=\frac{\theta_{\mathrm{p}}^{2}}{C_{\mathrm{p}}^{S}}=\frac{\bar{\theta}_{\mathrm{p}}^{2}}{\bar{C}_{\mathrm{p}}^{S}}=\frac{d_{31}^{2} E_{\mathrm{p}}}{\varepsilon_{33}^{T}-d_{31}^{2} E_{\mathrm{p}}} k_{\mathrm{p}} .
$$

Equation (16) implies that the stiffness of the electrical part is proportional to the stiffness of the mechanical part. The original and equivalent mechanical models, when electrical impedance $Z_{\mathrm{e}}$ is coupled to the piezoelectric element, are shown in Fig. 3. The electrical impedance $Z_{\mathrm{e}}$ is shown as the mechanical impedance $\bar{Z}_{\mathrm{e}}$ in the imaginary equivalent mechanical model and transformed into the mechanical impedance $Z_{\mathrm{m}}$ in the perfect

(a)

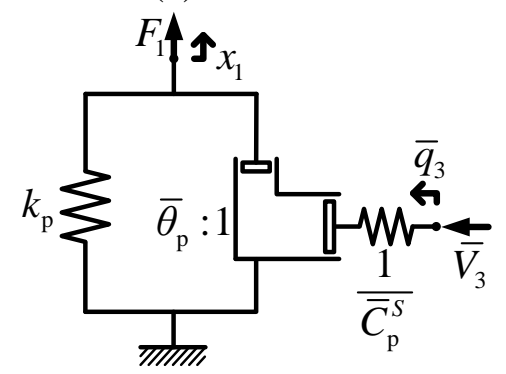

(b)

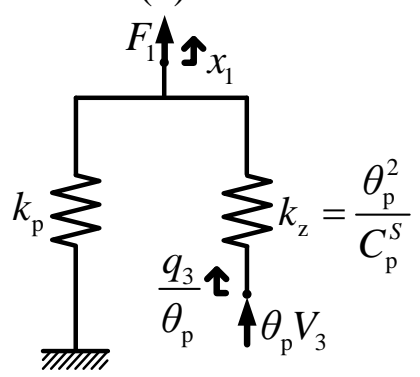

Fig. 2. Equivalent mechanical models of a piezoelectric element: (a) imaginary model and (b) perfect model.

(a)

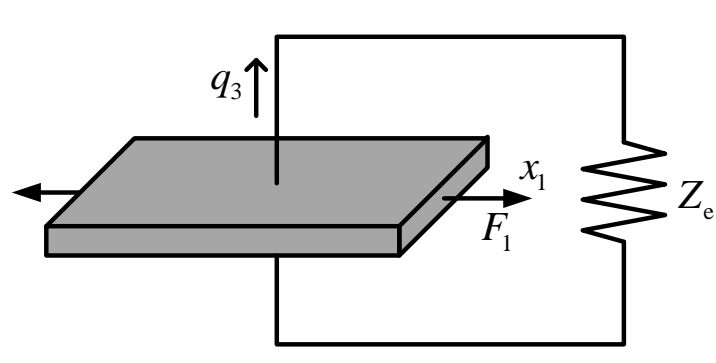

(b)

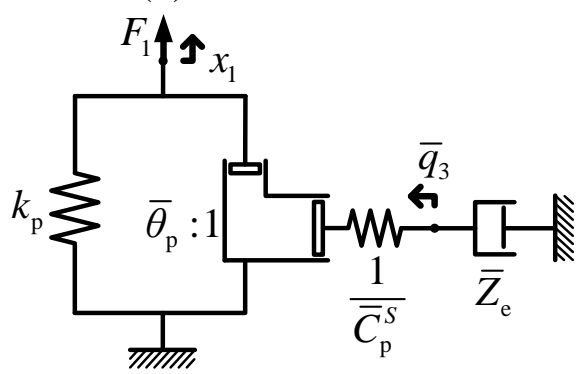

(c)

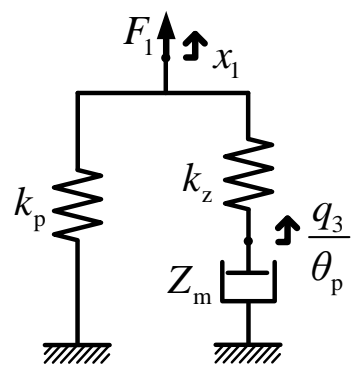

Fig. 3. Original and equivalent mechanical models of a piezoelectric element with electrical impedance: (a) original model, (b) imaginary model, and (c) perfect model. 
equivalent mechanical model. The mechanical impedance $Z_{\mathrm{m}}$ is written as follows.

$$
Z_{\mathrm{m}}=Z_{\mathrm{e}} \theta_{\mathrm{p}}^{2}=\bar{Z}_{\mathrm{e}} \bar{\theta}_{\mathrm{p}}^{2} \text {. }
$$

The lower end of the equivalent stiffness $k_{z}$ in Fig. 3 (b) is free when the electrodes of the piezoelectric element are shorted, and fixed when the electrodes are opened. When the electrodes are shorted, the electrical property of the piezoelectric element does not have any physical effect.

\subsection{Governing equations}

An example model of for application of vibration suppression using a piezoelectric element and electrical impedance $Z_{\mathrm{e}}$ is shown in Fig. 4. In this case, the cantilever is the host structure that is experiencing vibration suppression, and it is excited by external force $f_{\mathrm{e}}$. The piezoelectric element is attached to the host structure with an adhesive bond. Vibration suppression using a tuned LR circuit can suppress only a single vibration mode. Therefore the frequency range considered in this analysis is around the natural frequency of the targeted vibration mode, and the other vibration modes are ignored in theoretical analysis. In this case, their influences are small. The equation of motion when the electrodes of the piezoelectric element are shorted is written as follows.

$$
\begin{gathered}
M \ddot{\xi}+K \xi=B_{\mathrm{f}} f_{\mathrm{e}}, \\
M=M_{\mathrm{b}}+M_{\mathrm{p}}=1, \\
M_{\mathrm{b}}=\rho_{\mathrm{b}} w_{\mathrm{b}} t_{\mathrm{b}} \int_{0}^{l_{\mathrm{b}}} \psi^{2} d x, \\
M_{\mathrm{p}}=\rho_{\mathrm{p}} w_{\mathrm{p}} t_{\mathrm{p}} \int_{x_{\mathrm{f}}}^{x_{\mathrm{r}}} \psi^{2} d x, \\
K=K_{\mathrm{b}}+K_{\mathrm{p}}, \\
d z d x+\int_{x_{\mathrm{f}}}^{x_{\mathrm{r}}} \int_{-t_{\mathrm{b}}-t_{\mathrm{n}}}^{t_{\mathrm{n}}} z^{2}\left(\frac{\partial^{2} \psi}{\partial x^{2}}\right)^{2} d z d \\
=E_{\mathrm{p}} w_{\mathrm{p}} \int_{x_{\mathrm{l}}}^{x_{\mathrm{r}}} \int_{t_{\mathrm{n}}}^{t_{\mathrm{n}}+t_{\mathrm{p}}} z^{2}\left(\frac{\partial^{2} \psi}{\partial x^{2}}\right)^{2} d z d x \\
B_{\mathrm{f}}=\psi\left(x_{\mathrm{f}}\right), \\
t_{\mathrm{n}}=\frac{E_{\mathrm{b}} w_{\mathrm{b}} t_{\mathrm{b}}^{2}-E_{\mathrm{p}} w_{\mathrm{p}} t_{\mathrm{p}}^{2}}{2\left(E_{\mathrm{b}} w_{\mathrm{b}} t_{\mathrm{b}}+E_{\mathrm{p}} w_{\mathrm{p}} t_{\mathrm{p}}\right)},
\end{gathered}
$$

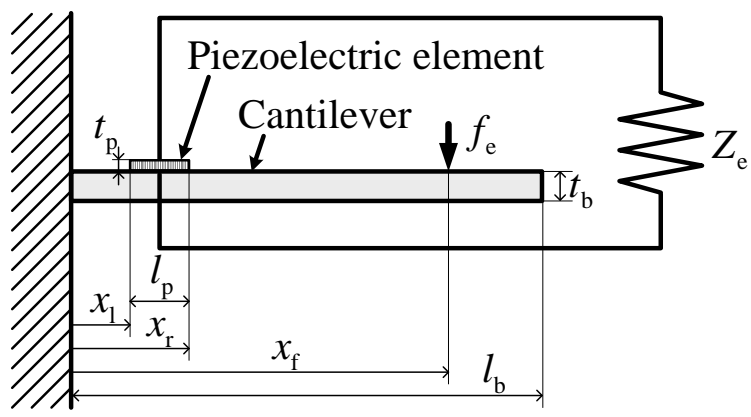

Fig. 4. A model of vibration suppression using a piezoelectric element and electrical impedance. 
where $\xi$ is the modal displacement, $\rho_{\mathrm{b}}$ and $\rho_{\mathrm{p}}$ are the densities of the beam and the piezoelectric element, respectively, $E_{\mathrm{b}}$ is the Young's modulus of the beam, $l_{\mathrm{b}}, w_{\mathrm{b}}, t_{\mathrm{b}}$ are the length, width, and thickness of the beam, respectively, $t_{\mathrm{n}}$ is the distance between the neutral axis and the adverse side of the beam within the range where the piezoelectric element is attached, $x_{1}, x_{\mathrm{r}}, x_{\mathrm{f}}$ are the distance between the clamped end and the left and right endpoints of the piezoelectric element and the point where the external force is added, respectively, $\psi$ is the shape function of the targeted vibration mode. The $x$ axis is the longitudinal direction and the origin is at the clamped end. The variable $z$ denotes the distance from the neutral axis. Since this cantilever is thin, the shear deformation and rotary inertia of the cantilever are ignored. As written in Eq. (19), the shape function $\psi$ used in this paper is normalized as the modal mass $M$ becomes 1 . When the electrodes are shorted, the electrical property of the piezoelectric element does not affect the system. The equation of motion (18) is derived from the simple mechanical model shown in Fig. 5. Here the cylinder is fixed in space, and the area ratio $\theta_{\mathrm{k}}$ is written as

$$
\theta_{\mathrm{k}}=\sqrt{\frac{K_{\mathrm{p}}}{k_{\mathrm{p}}}} .
$$

Because $k_{\mathrm{p}}$ and $k_{\mathrm{z}}$ are in the proportional relation from Eq. (16), the imaginary and perfect equivalent mechanical models when the electrodes of the piezoelectric element are shunted by the electrical impedance $Z_{\mathrm{e}}$ are drawn as Fig. 6. From these equivalent mechanical models, the governing equations are written as follows.

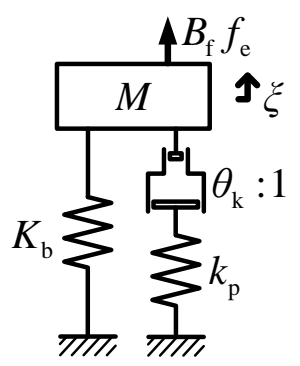

Fig. 5. Equivalent mechanical model of the targeted vibration mode with a short-circuited piezoelectric element.

(a)

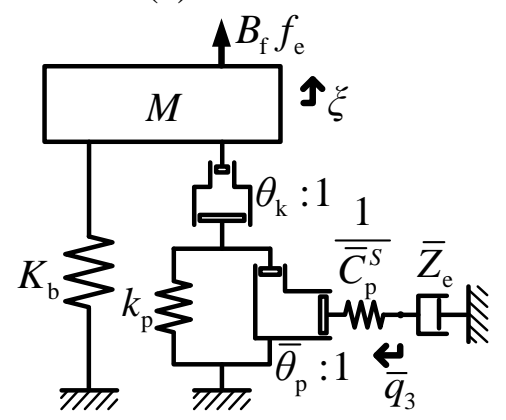

(b)

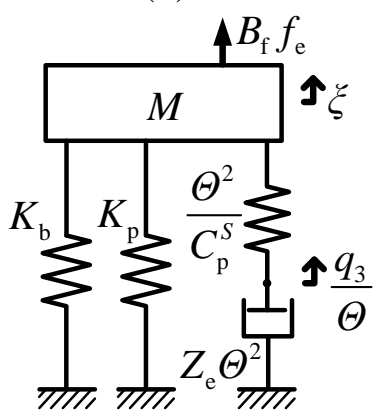

Fig. 6. Equivalent mechanical models of the targeted vibration mode with a piezoelectric element shunted by electrical impedance $Z_{\mathrm{e}}$ : (a) imaginary model and (b) perfect model. 


$$
\begin{gathered}
M \ddot{\xi}+K \xi+\Theta\left(\frac{\Theta}{C_{\mathrm{p}}^{S}} \xi-\frac{1}{C_{\mathrm{p}}^{S}} q_{3}\right)=B_{\mathrm{f}} f_{\mathrm{e}}, \\
Z_{\mathrm{e}} \dot{q}_{3}+\frac{1}{C_{\mathrm{p}}^{S}} q_{3}=\frac{\Theta}{C_{\mathrm{p}}^{S}} \xi
\end{gathered}
$$

where $\Theta$ is the modal electromechanical coupling coefficient, and given as follows.

$$
\Theta=\theta_{\mathrm{p}} \theta_{\mathrm{k}} \text {. }
$$

\subsection{Passive vibration suppression using a LR circuit}

Models for passive vibration suppression using series and parallel LR circuits are shown in Fig. 7. Electrical impedances of the series and parallel LR circuits are written as

$$
Z_{\mathrm{e}}= \begin{cases}j \omega L+R & (\text { series }) \\ \frac{j \omega L R}{j \omega L+R} & (\text { parallel })\end{cases}
$$

where $j$ is the imaginary unit, $\omega$ is the excitation frequency, $L$ is the inductance, and $R$ is the resistance. The perfect equivalent mechanical models are depicted in Fig. 8. Compared to a typical mechanical vibration absorber, the positions of the dashpots are different. From Eqs. (28), (29) and (31), the nondimensional compliance is derived as

$$
\begin{gathered}
\frac{\Xi}{\Xi_{\mathrm{st}}}= \begin{cases}\frac{1}{-g^{2}+1+\beta G_{\mathrm{S}}} & (\text { series }) \\
\frac{1}{-g^{2}+1+\beta G_{\mathrm{P}}} & \text { (parallel) }\end{cases} \\
G_{\mathrm{S}}=\frac{-g^{2}+2 j \zeta_{\mathrm{S}} f g}{f^{2}-g^{2}+2 j \zeta_{\mathrm{S}} f g}, \\
G_{\mathrm{P}}=\frac{-g^{2}}{f^{2}-g^{2}+2 j \zeta_{\mathrm{P}} f g},
\end{gathered}
$$

(a)

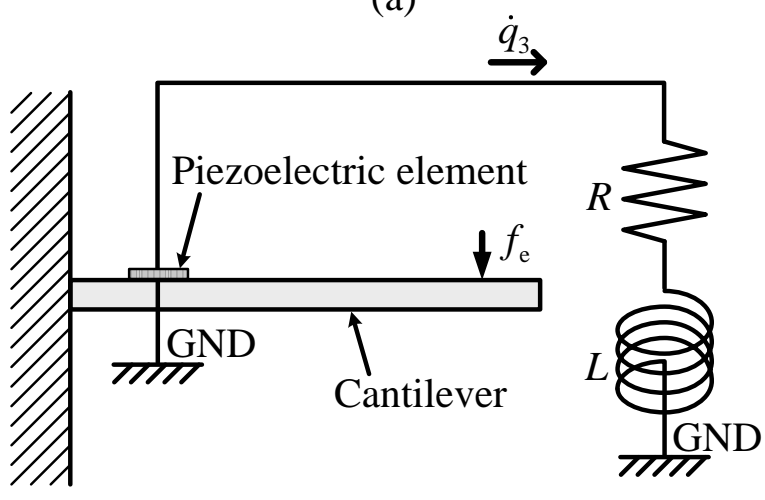

(b)

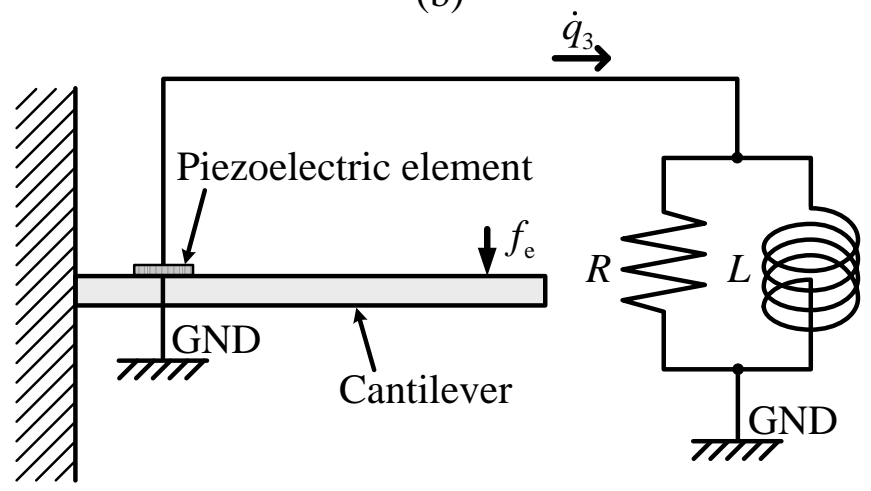

Fig. 7. Schematic diagrams of passive vibration suppression using series and parallel LR circuits: (a) series LR circuit and (b) parallel LR circuit. 
(a)

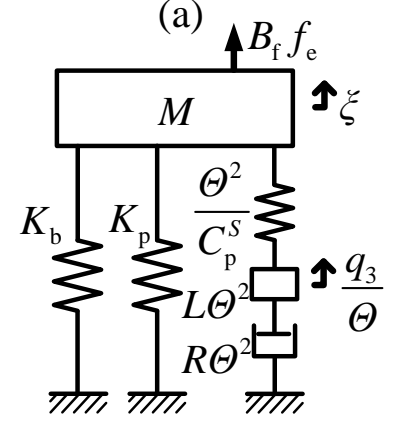

(b)

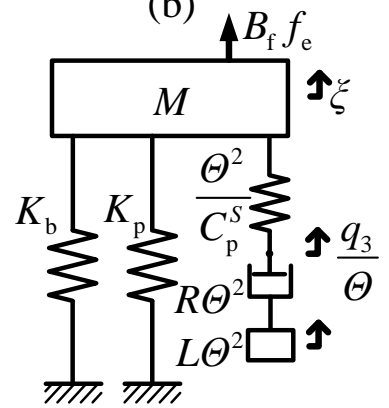

Fig. 8. Perfect equivalent mechanical models of passive vibration suppression using series and parallel LR circuits: (a) series LR circuit and (b) parallel LR circuit.

$$
\begin{gathered}
\Xi_{\mathrm{st}}=\frac{B_{\mathrm{f}} F_{\mathrm{e}}}{K}, \quad g=\frac{\omega}{\Omega}, \quad \beta=\frac{\Theta^{2}}{K C_{\mathrm{p}}^{S}}, \\
f=\frac{\omega_{\mathrm{a}}}{\Omega}, \quad \zeta_{\mathrm{S}}=\frac{R}{2} \sqrt{\frac{C_{\mathrm{p}}^{S}}{L}}, \quad \zeta_{\mathrm{P}}=\frac{1}{2 R} \sqrt{\frac{L}{C_{\mathrm{p}}^{S}}}, \quad \Omega=\sqrt{\frac{K}{M}}, \omega_{\mathrm{a}}=\sqrt{\frac{1}{L C_{\mathrm{p}}^{S}}},
\end{gathered}
$$

Here $\Xi$ and $F_{\mathrm{e}}$ are the complex amplitude of $\xi$ and $f_{\mathrm{e}}$, respectively. From Eq. (32), the magnitudes of the nondimensional compliance $u_{\mathrm{C}}$, mobility $u_{\mathrm{M}}$, and accelerance $u_{\mathrm{A}}$ are given as follows.

$$
\begin{aligned}
& u_{\mathrm{C}}=\left|\frac{\Xi}{\Xi_{\mathrm{st}}}\right|=\left\{\begin{array}{ll}
\sqrt{\frac{a^{2}+4 \zeta_{\mathrm{S}}^{2} b^{2}}{c^{2}+4 \zeta_{\mathrm{S}}^{2} d_{\mathrm{S}}^{2}}} & (\text { series }) \\
\sqrt{\frac{a^{2}+4 \zeta_{\mathrm{P}}^{2} b^{2}}{c^{2}+4 \zeta_{\mathrm{P}}^{2} d_{\mathrm{P}}^{2}}} & (\text { parallel })
\end{array},\right. \\
& u_{\mathrm{M}}=\frac{1}{\Omega}\left|\frac{\dot{\Xi}}{\Xi_{\mathrm{st}}}\right|=\left\{\begin{array}{ll}
\sqrt{\frac{g^{2}\left(a^{2}+4 \zeta_{\mathrm{S}}^{2} b^{2}\right)}{c^{2}+4 \zeta_{\mathrm{S}}^{2} d_{\mathrm{S}}^{2}}} & \text { (series) } \\
\sqrt{\frac{g^{2}\left(a^{2}+4 \zeta_{\mathrm{P}}^{2} b^{2}\right)}{c^{2}+4 \zeta_{\mathrm{P}}^{2} d_{\mathrm{P}}^{2}}} & \text { (parallel) }
\end{array}\right. \text {, } \\
& u_{\mathrm{A}}=\frac{1}{\Omega^{2}}\left|\frac{\ddot{\Xi}}{\Xi_{\mathrm{st}}}\right|=\left\{\begin{array}{ll}
\sqrt{\frac{g^{4}\left(a^{2}+4 \zeta_{\mathrm{S}}^{2} b^{2}\right)}{c^{2}+4 \zeta_{\mathrm{S}}^{2} d_{\mathrm{S}}^{2}}} & \text { (series) } \\
\sqrt{\frac{g^{4}\left(a^{2}+4 \zeta_{\mathrm{P}}^{2} b^{2}\right)}{c^{2}+4 \zeta_{\mathrm{P}}^{2} d_{\mathrm{P}}^{2}}} & \text { (parallel) }
\end{array},\right. \\
& a=f^{2}-g^{2} \text {, } \\
& b=f g \text {, } \\
& c=\left(1-g^{2}\right)\left(f^{2}-g^{2}\right)-\beta g^{2}, \\
& d_{\mathrm{S}}=f g\left(1-g^{2}+\beta\right) \text {, } \\
& d_{\mathrm{P}}=f g\left(1-g^{2}\right) \text {, } \\
& \dot{\Xi}=j \omega \Xi \text {, }
\end{aligned}
$$




$$
\ddot{\Xi}=-\omega^{2} \Xi \text {. }
$$

\subsubsection{Optimum tuning in compliance}

The two fixed points method [12] is common for finding the optimum natural frequency ratio and the resistance ratio of the additional one degree of freedom system that minimizes the maximum amplitude in the frequency domain. The two fixed points method is often used in optimum tuning of mechanical vibration absorbers because of its simplicity; it is also applied to the optimum tuning of the series and parallel LR circuits in this paper.

Because the magnitude of the nondimensional compliance (43) has two fixed points that are independent of the resistance ratio, the optimum natural frequency ratio is determined so that amplitudes at the two fixed points become equal, and the optimum resistance ratio is derived so that amplitude is maximized at the two fixed points.

The optimum natural frequency ratio in the magnitude of the nondimensional compliance (43) is given for the condition that the amplitudes at two fixed points—given by A and B-are equal.

$$
f_{\text {Copt }}=\left\{\begin{array}{ll}
\sqrt{1+\beta} & \text { (series }) \\
\sqrt{\frac{2-\beta}{2}} & \text { (parallel) }
\end{array} .\right.
$$

The nondimensional frequencies of the two fixed points are given as

$$
g_{\mathrm{A}, \mathrm{B}}=\left\{\begin{array}{ll}
\sqrt{(1+\beta) \mp \sqrt{\frac{\beta(1+\beta)}{2}}} & \text { (series) } \\
\sqrt{1 \mp \sqrt{\frac{\beta}{2}}} & \text { (parallel) }
\end{array} .\right.
$$

The amplitudes at the two fixed points are derived as follows.

$$
\left.u_{\mathrm{C}}\right|_{g=g_{\mathrm{A}, \mathrm{B}}}=\left\{\begin{array}{ll}
\sqrt{\frac{2}{\beta(1+\beta)}} & \text { (series) } \\
\sqrt{\frac{2}{\beta}} & \text { (parallel) }
\end{array} .\right.
$$

The optimum resistance ratios at the two fixed points are given as

$$
\begin{cases}\zeta_{\mathrm{SA}, \mathrm{B}}=\left.\frac{1}{2} \sqrt{\frac{-a a^{\prime}+u_{\mathrm{C}}^{2} c c^{\prime}}{b b^{\prime}-u_{\mathrm{C}}^{2} d_{\mathrm{S}} d_{\mathrm{S}}^{\prime}}}\right|_{g=g_{\mathrm{A}, \mathrm{B}}} \quad(\text { series }) \\ \zeta_{\mathrm{PA}, \mathrm{B}}=\left.\frac{1}{2} \sqrt{\frac{-a a^{\prime}+u_{\mathrm{C}}^{2} c c^{\prime}}{b b^{\prime}-u_{\mathrm{C}}^{2} d_{\mathrm{P}} d_{\mathrm{P}}^{\prime}}}\right|_{g=g_{\mathrm{A}, \mathrm{B}}} \quad \text { (parallel) }\end{cases}
$$

where the prime' denotes $\partial / \partial g . \zeta_{\mathrm{A}}$ and $\zeta_{\mathrm{B}}$ are not equal; however, the difference is minute. The arithmetic average, geometric average, and root mean square of $\zeta_{\mathrm{A}}$ and $\zeta_{\mathrm{B}}$ can all be used as the optimum resistance ratio because the difference between them is small enough to be ignored. In this paper, the optimum 
resistance ratio is defined by root mean square because of simplicity of the expression.

$$
\left\{\begin{array}{l}
\zeta_{\mathrm{CSopt}}=\sqrt{\frac{\zeta_{\mathrm{SA}}^{2}+\zeta_{\mathrm{SB}}^{2}}{2}}=\frac{1}{2} \sqrt{\frac{3 \beta}{2+\beta}} \quad(\text { series }) \\
\zeta_{\mathrm{CPopt}}=\sqrt{\frac{\zeta_{\mathrm{PA}}^{2}+\zeta_{\mathrm{PB}}^{2}}{2}}=\frac{1}{2} \sqrt{\frac{3 \beta}{2-\beta}} \quad \text { (parallel) }
\end{array}\right.
$$

As indicated by Eqs. (53) and (57), both optimum natural frequency ratio and optimum resistance ratio are determined only by the equivalent stiffness ratio $\beta$.

\subsubsection{Optimum tuning in mobility}

Optimum values of the series and parallel LR circuits in terms of the magnitude of the nondimensional mobility can be derived by the two fixed points method as well as in terms of the magnitude of the nondimensional compliance. The optimum natural frequency ratio, the nondimensional frequencies of the two fixed points, the amplitudes at the two fixed points, and the optimum resistance ratio are given as follows.

$$
\begin{gathered}
f_{\mathrm{Mopt}}=\left\{\begin{array}{ll}
(1+\beta) \sqrt{\frac{2}{2+\beta}} & (\text { series }) \\
1 & (\text { parallel })
\end{array},\right. \\
g_{\mathrm{A}, \mathrm{B}}=\left\{\begin{array}{ll}
\sqrt{\frac{(1+\beta)[(4+3 \beta) \mp \sqrt{\beta(8+5 \beta)}]}{2(2+\beta)}} \quad \text { (series) } \\
\sqrt{\frac{(4+\beta) \mp \sqrt{\beta(8+\beta)}}{4}} \quad \text { (parallel) }
\end{array},\right. \\
\left.u_{\mathrm{M}}\right|_{g=g_{\mathrm{A}, \mathrm{B}}}= \begin{cases}\sqrt{\frac{2+\beta}{\beta(1+\beta)}} & \text { (series) } \\
\zeta_{\mathrm{MSopt}}=\frac{1}{4} \sqrt{\frac{\beta\left(24+24 \beta+5 \beta^{2}\right)}{(1+\beta)(2+\beta)^{2}}} \quad \text { (series) } & \text { (parallel) } \\
\zeta_{\mathrm{MPopt}}=\frac{1}{2} \sqrt{\frac{3 \beta}{2}} \quad \text { (parallel) }\end{cases}
\end{gathered}
$$

\subsubsection{Optimum tuning in accelerance}

Optimum values of the series and parallel LR circuits for the magnitude of the nondimensional accelerance can be derived by the two fixed points method as well as for the magnitude of the nondimensional compliance. The optimum natural frequency ratio, the nondimensional frequencies of the two fixed points, the amplitudes at the two fixed points, and the optimum resistance ratio are given as follows. 


$$
\begin{aligned}
& f_{\text {A opt }}= \begin{cases}1+\beta & (\text { series }) \\
\sqrt{\frac{2+\beta}{2}} & (\text { parallel })\end{cases} \\
& g_{\mathrm{A}, \mathrm{B}}=\left\{\begin{array}{ll}
\sqrt{\frac{(1+\beta)[(2+\beta) \mp \sqrt{\beta(2+\beta)}]}{2}} & \text { (series) } \\
\sqrt{\frac{(2+\beta) \mp \sqrt{\beta(2+\beta)}}{2}} & \text { (parallel) }
\end{array},\right. \\
& \left.u_{\mathrm{A}}\right|_{g=g_{\mathrm{A}, \mathrm{B}}}=\left\{\begin{array}{ll}
\sqrt{\frac{2+\beta}{\beta}} & (\text { series }) \\
\sqrt{\frac{2+\beta}{\beta}} & (\text { parallel })
\end{array},\right. \\
& \left\{\begin{array}{ll}
\zeta_{\mathrm{ASopt}}=\frac{1}{2} \sqrt{\frac{3 \beta}{2(1+\beta)}} & (\text { series }) \\
\zeta_{\mathrm{APopt}}=\frac{1}{2} \sqrt{\frac{3 \beta}{2}} & \text { (parallel) }
\end{array} .\right.
\end{aligned}
$$

\subsubsection{Optimum values of the inductance and the resistance}

Using the optimum natural frequency ratio $f_{\mathrm{opt}}$ and the optimum resistance ratios $\zeta_{\text {Sopt }}$ and $\zeta_{\text {Popt }}$, the optimum values of the inductance and the resistance are formulated as follows.

$$
\begin{gathered}
L_{\mathrm{opt}}=\frac{1}{f_{\mathrm{opt}}^{2}} \frac{1}{C_{\mathrm{p}}^{S}} \frac{1}{\Omega^{2}}, \\
R_{\mathrm{opt}}= \begin{cases}2 \zeta_{\mathrm{Sopt}} \frac{1}{f_{\mathrm{opt}}} \frac{1}{C_{\mathrm{p}}^{S}} \frac{1}{\Omega} & \text { (series) } \\
\frac{1}{2 \zeta_{\mathrm{Popt}}} \frac{1}{f_{\mathrm{opt}}} \frac{1}{C_{\mathrm{p}}^{S}} \frac{1}{\Omega} & \text { (parallel) }\end{cases}
\end{gathered}
$$

\subsection{Comparison between series and parallel LR circuits}

\subsubsection{Performance comparison}

The vibration suppression performance is evaluated based on the amplitude at the two fixed points because the amplitude at these points is maximized. The amplitudes at the two fixed points for the compliance, mobility, and accelerance are given by Eqs. (55), (60), and (64), respectively. The amplitudes are evaluated by using only the equivalent stiffness ratio $\beta$ as an independent variable. The relationship between the amplitude at the two fixed points and the equivalent stiffness ratio $\beta$ is shown in Fig. 9. The amplitude of a series LR circuit is smaller than that of a parallel LR circuit for the compliance and mobility, and they are equal in the accelerance. The amplitudes of series and parallel LR circuits are almost equal when the value of the equivalent stiffness ratio 

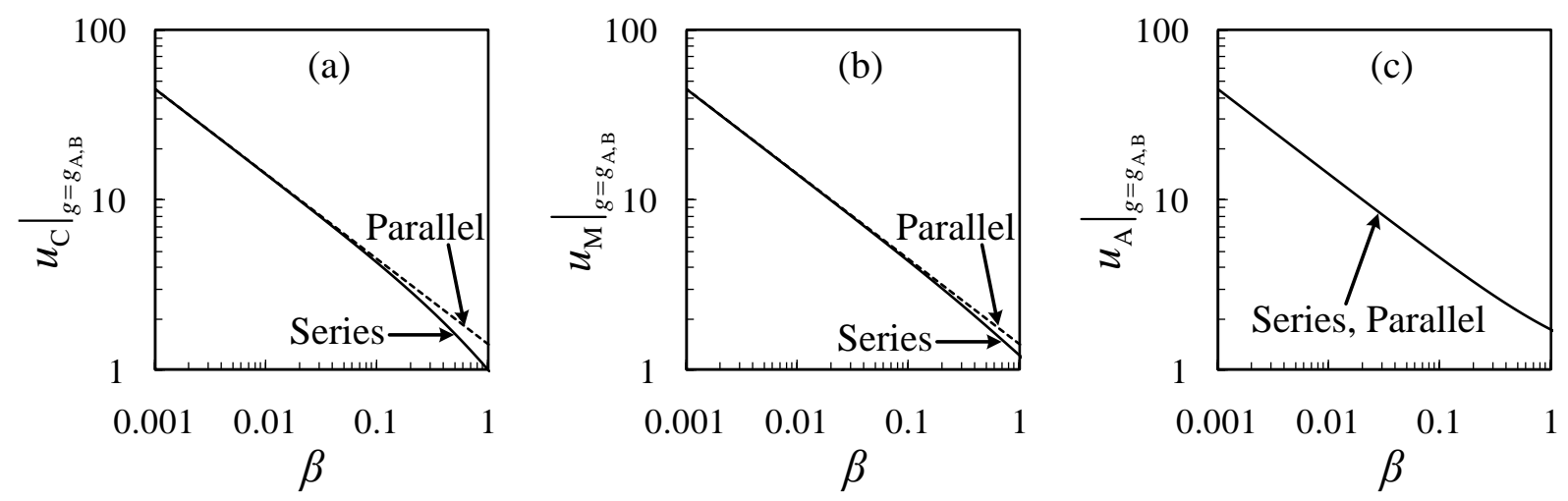

Fig. 9. The relationship between the amplitude at the two fixed points and the equivalent stiffness ratio: (a) in compliance, (b) in mobility, and (c) in accelerance.

$\beta$ is much smaller than 1.

The performance of a typical mechanical vibration absorber is evaluated based on the mass ratio. By contrast, the performance of vibration suppression using piezoelectric elements uses the stiffness ratio because the electrical properties of a piezoelectric element correspond to a spring in the equivalent mechanical model. However, the mass ratio can also be used in vibration suppression with piezoelectric elements and LR circuits. From Eqs. (37), (41), and (66), the equivalent mass ratio is given as follows.

$$
\mu=\frac{L \Theta^{2}}{M}=\frac{\beta}{f_{\mathrm{opt}}^{2}} .
$$

The optimum natural frequency ratio $f_{\mathrm{opt}}$ approaches 1 , and so the values of the mass and stiffness ratios are nearly equal. The performances of mechanical and electrical vibration absorbers can be compared by using the mass and stiffness ratios.

From Eqs. (32)-(34), the stiffness and damping added by the additional electrical system are evaluated by $\beta G_{\mathrm{S}}$ and $\beta G_{\mathrm{P}}$. This paper defines added stiffness ratio $\gamma_{K}$ and added damping ratio $\gamma_{D}$ as follows.

$$
\begin{aligned}
& \gamma_{K}= \begin{cases}\operatorname{Re}\left(\beta G_{\mathrm{S}}\right) & (\text { series }) \\
\operatorname{Re}\left(\beta G_{\mathrm{P}}\right) & (\text { parallel })\end{cases} \\
& \gamma_{D}= \begin{cases}\frac{\operatorname{Im}\left(\beta G_{\mathrm{S}}\right)}{2 g} & (\text { series }) \\
\frac{\operatorname{Im}\left(\beta G_{\mathrm{P}}\right)}{2 g} & (\text { parallel })\end{cases}
\end{aligned}
$$

As an example, $\gamma_{K}$ and $\gamma_{D}$ with $\beta=0.030$ are shown in Fig. 10. In this example, optimum values of LR circuits in the compliance were adopted. The results of a typical mechanical vibration absorber (MVA) using the same stiffness ratio are also shown in Fig. 10. Around the natural frequency, the added stiffness ratio of a series LR circuit is larger than that of a parallel LR circuit, and the added damping ratios are nearly equal. These are the reasons that the performance of a series LR circuit is small degree better than that of a parallel LR circuit in terms of the compliance and mobility. The added stiffness ratios approach $\beta$ when $g$ becomes large. The 

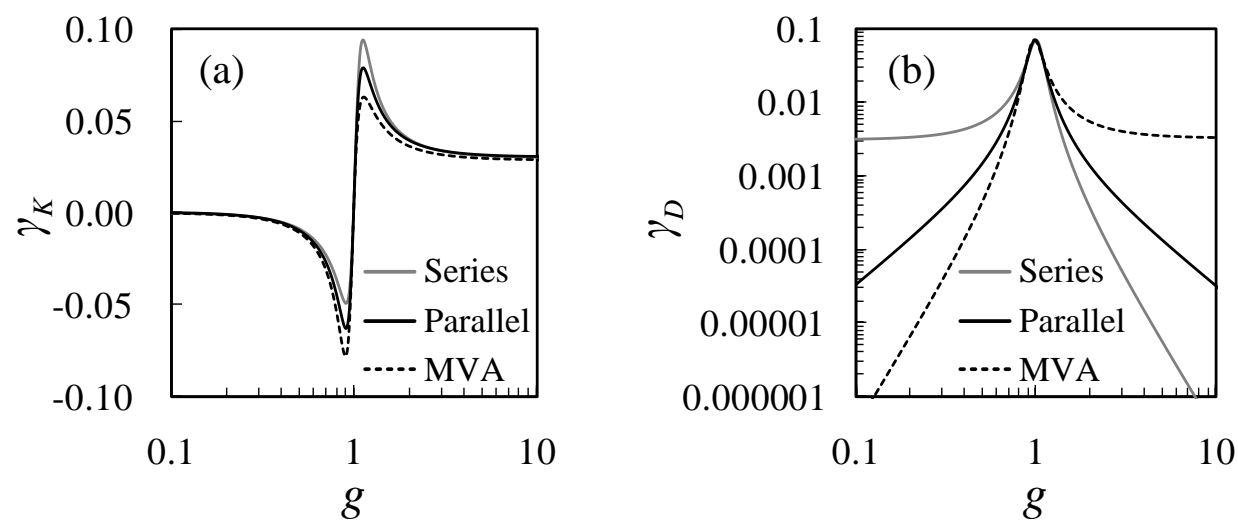

Fig. 10. Frequency characteristics of the added stiffness and damping ratios of series and parallel LR circuits and a typical mechanical vibration absorber using $\beta=0.030$ : (a) added stiffness ratio and (b) added damping ratio.

added damping ratio of a series LR circuit approach $\beta \zeta_{\text {sopt }} / f_{\text {opt }}$ when $g$ becomes small. A series LR circuit gives damping to the main system in the frequency range less than the natural frequency.

\subsubsection{Comparison of optimum values of inductance}

Inductance should be tuned so that the system has an electrical resonance. In this case, the resonance frequency of the electrical system is nearly equal to the natural frequency of the main system. This represents an optimal condition. Because the added stiffness of a series LR circuit is larger than that of a parallel LR circuit, as shown in Fig. 10 (a), the optimum natural frequency ratio of a series LR circuit is larger than that of a parallel LR circuit. As a result, the optimum values of the inductance of a series LR circuit are smaller; however, the difference is usually small because of the smallness of the equivalent stiffness ratio $\beta$.

\subsubsection{Comparison of optimum values of resistance}

In general, the equivalent stiffness ratio is much smaller than 1. In this case, the ratio of optimum values of the resistances is given as

$$
R_{\text {Sopt }}: R_{\text {Popt }}=2 \zeta_{\text {Sopt }} \frac{1}{f_{\text {Sopt }}} \frac{1}{C_{\mathrm{p}}^{S}} \frac{1}{\Omega}: \frac{1}{2 \zeta_{\text {Popt }}} \frac{1}{f_{\text {Popt }}} \frac{1}{C_{\mathrm{p}}^{S}} \frac{1}{\Omega} \approx 4 \zeta_{\text {Sopt }} \zeta_{\text {Popt }}: 1,
$$

where $R_{\text {Sopt }}$ and $R_{\text {Popt }}$ are the optimum resistances of series and parallel LR circuits, respectively, and $f_{\text {Sopt }}$ and $f_{\text {Popt }}$ are the optimum natural frequency ratios of series and parallel LR circuits, respectively. $\zeta_{\text {Sopt }}$ and $\zeta_{\text {Popt }}$ are given by Eqs. (57), (61), and (65), and they are usually much smaller than 1; therefore, $R_{\mathrm{Popt}}$ is much larger than $R_{\text {Sopt }}$.

Since the electrical resonance of the additional electrical system suppresses vibration of the host structure, the current which flows back and forth between the inductance and the capacitance should be large. In the method using a series LR circuit, the amplitude of electrical charge becomes large if the resistance is small. By contrast, the amplitude of electrical charge for the method using a parallel LR circuit becomes large if the resistance is large. These are the reasons that $R_{\mathrm{Popt}}$ is much larger than $R_{\text {Sopt }}$. 


\subsubsection{Summary of comparison}

A series LR circuit is usually superior to a parallel LR circuit in terms of performance; however, there are some exceptions. When the value of $C_{\mathrm{p}}^{S}$ is large and $R_{\mathrm{Sopt}}$ is small, the performance is greatly decreased because of the variation in the value of the resistance. In other words, the performance of a parallel LR circuit is more robust than that of a series LR circuit because $R_{\mathrm{Popt}}$ is very large. The same thing is adopted for the value of $\Omega$. The circuit should be chosen in consideration of not only performance but also robustness.

\subsection{Dielectric loss of a piezoelectric element and internal resistance of an inductor}

In practice piezoelectric elements dissipate some energy due to dielectric loss. This phenomenon is caused by the relaxation time of polarization. As shown in Fig. 11 (a), the dielectric loss can be expressed by a parallel resistance $R_{C}$ in the equivalent circuit. $R_{C}$ is given as follows.

$$
R_{C}=\frac{1}{\omega C_{\mathrm{p}}^{T} \tan \delta},
$$

where $\delta$ is the dielectric loss factor. When $R_{C}=\infty$, there is no energy loss.

Similarly, inductors also dissipate energy because they have not only inductance but also internal resistance. As shown in Fig. 11 (b), the internal resistance can be expressed by a series resistance $R_{L}$.

If the dissipated energy due to $R_{C}$ and $R_{L}$ is very small, they are negligible. However, sometimes the influence of $R_{C}$ and $R_{L}$ cannot be ignored. The models of passive vibration suppression, including $R_{C}$ and $R_{L}$, are shown in Fig. 12. The influence of $R_{C}$ and $R_{L}$ is identical to the resistances in the parallel and series LR circuits, respectively. The nondimensional compliance then is given as

$$
\begin{gathered}
\frac{\Xi}{\Xi_{\mathrm{st}}}=\left\{\begin{array}{cc}
\frac{1}{-g^{2}+1+\beta G_{\mathrm{S} 2}} & (\text { series }) \\
\frac{1}{-g^{2}+1+\beta G_{\mathrm{P} 2}} & (\text { parallel })
\end{array}\right. \\
G_{\mathrm{S} 2}=\frac{-g^{2}+2 j \zeta_{1} f g}{\left(1+4 \zeta_{1} \zeta_{C}\right) f^{2}-g^{2}+2 j\left(\zeta_{1}+\zeta_{C}\right) f g},
\end{gathered}
$$

(a)

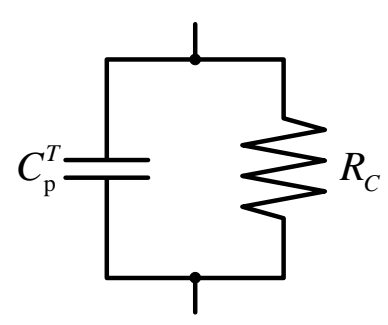

(b)

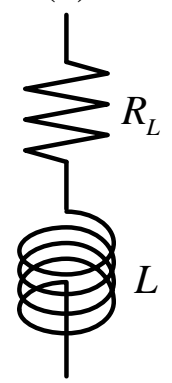

Fig. 11. Equivalent circuit including the dielectric loss of the piezoelectric element and the internal resistance in the inductor: (a) dielectric loss of the piezoelectric element and (b) internal resistance in the inductor. 

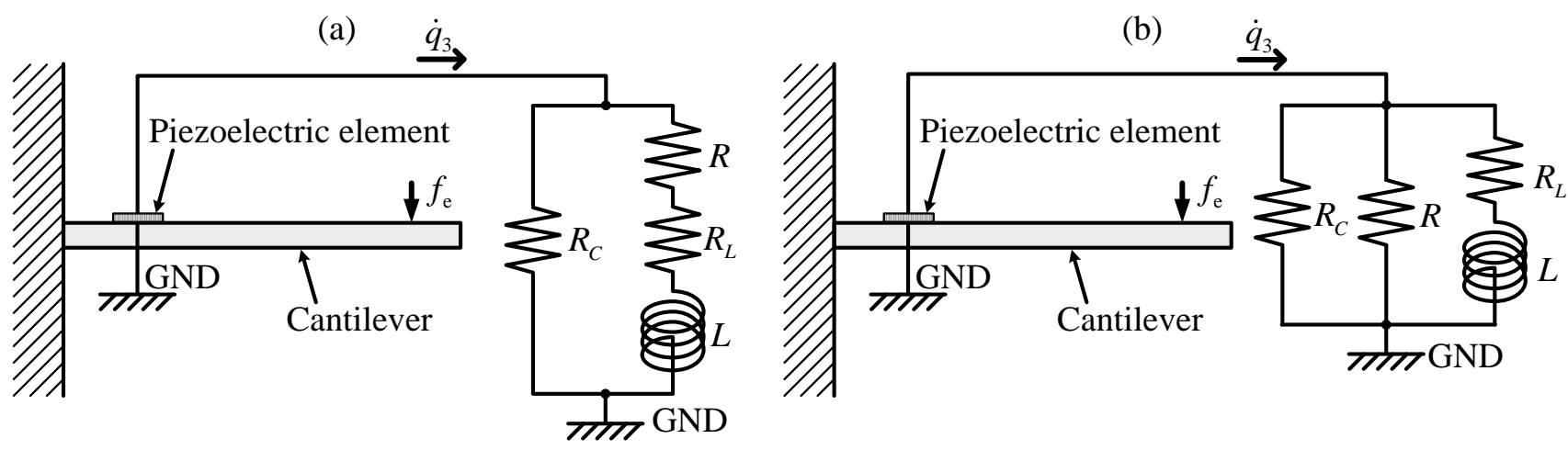

Fig. 12. Schematic diagrams of passive vibration suppression including $R_{C}$ and $R_{L}$ : (a) series LR circuit and (b) parallel LR circuit.

$$
\begin{gathered}
G_{\mathrm{P} 2}=\frac{-g^{2}+2 j \zeta_{L} f g}{\left(1+4 \zeta_{L} \zeta_{2}\right) f^{2}-g^{2}+2 j\left(\zeta_{L}+\zeta_{2}\right) f g} \\
\zeta_{C}=\frac{1}{2 R_{C}} \sqrt{\frac{L}{C_{\mathrm{p}}^{S}}}, \zeta_{L}=\frac{R_{L}}{2} \sqrt{\frac{C_{\mathrm{p}}^{S}}{L}}, \zeta_{1}=\zeta_{\mathrm{S}}+\zeta_{L}, \quad \zeta_{2}=\zeta_{\mathrm{P}}+\zeta_{C}
\end{gathered}
$$

In this case, it is impossible to derive the optimum values of the circuit theoretically by use of the two fixed points method because there are no fixed points in Eq. (73). However, the values can be estimated approximately. The practical models are intermediates between the two ideal models shown in Fig. 7. Therefore, the optimum values in the practical models are expected to be close to the optimum values in the ideal models. From Eqs. (53), (58), and (62), the optimum natural frequency ratios in the practical models are expected to approach 1 . The total resistance ratios in the practical models are defined as follows.

$$
\zeta_{\mathrm{T}}=\left\{\begin{array}{ll}
\zeta_{\mathrm{S}}+\zeta_{L}+\zeta_{C} & (\text { series }) \\
\zeta_{\mathrm{P}}+\zeta_{L}+\zeta_{C} & (\text { parallel })
\end{array} .\right.
$$

From Eqs. (57), (61), and (65), the optimum resistance ratios in the ideal models are almost equal although the values of the resistances are significantly different. It suggests that the optimum resistance ratio does not depend on the position of the resistance. Therefore, the total resistance ratios in the practical models should be tuned to be close to the optimum resistance ratios in the ideal models. The value of the resistance, which should be used in the experiment, can be estimated when the resistance ratios $\zeta_{C}$ and $\zeta_{L}$ are given. If $\zeta_{C}+\zeta_{L}$ is larger than the optimum resistance ratio, LR circuits can not be optimally tuned. Therefore, piezoelectric elements and inductors with small resistance ratios should be chosen.

\subsection{Effect of stiffness of adhesive bond}

Piezoelectric elements are attached to the target with adhesive bonds. In the preceding subsections, piezoelectric elements were assumed to be fixed to the host structure, and the effect of the adhesive bond was ignored. However, the stiffness of the adhesive bond is generally not large enough to be ignored. The elemental imaginary and perfect equivalent mechanical models including the stiffness of adhesive bonds are shown in Fig. 
13. Here $k_{\mathrm{a} 1}$ is the stiffness of the adhesive bond, and $x_{\mathrm{a} 1}$ is the displacement of the connecting point between the adhesive bond and the piezoelectric element. From these equivalent mechanical models, the equilibria of force are given as follows.

$$
\begin{gathered}
F_{1}=k_{\mathrm{a} 1}\left(x_{1}-x_{\mathrm{a} 1}\right), \\
k_{\mathrm{a} 1}\left(x_{1}-x_{\mathrm{a} 1}\right)=k_{\mathrm{p}} x_{\mathrm{a} 1}+k_{\mathrm{z}}\left(x_{\mathrm{a} 1}-\frac{q_{3}}{\theta_{\mathrm{p}}}\right), \\
k_{\mathrm{z}}\left(x_{\mathrm{a} 1}-\frac{q_{3}}{\theta_{\mathrm{p}}}\right)=-\theta_{\mathrm{p}} V_{3},
\end{gathered}
$$

Equations (81)-(83) are equivalently transformed into the following equations.

$$
\begin{gathered}
F_{1}=k_{\mathrm{p}}^{\prime} x_{1}+k_{\mathrm{z}}^{\prime}\left(x_{1}-\frac{q_{3}}{\theta_{\mathrm{p}}^{\prime}}\right), \\
k_{\mathrm{z}}^{\prime}\left(x_{1}-\frac{q_{3}}{\theta_{\mathrm{p}}^{\prime}}\right)=-\theta_{\mathrm{p}}^{\prime} V_{3},
\end{gathered}
$$

where

$$
\begin{gathered}
k_{\mathrm{p}}^{\prime}=\frac{k_{\mathrm{a} 1}}{k_{\mathrm{a} 1}+k_{\mathrm{p}}} k_{\mathrm{p}}, \\
k_{\mathrm{z}}^{\prime}=\frac{k_{\mathrm{a} 1}^{2}}{\left(k_{\mathrm{a} 1}+k_{\mathrm{p}}+k_{\mathrm{z}}\right)\left(k_{\mathrm{a} 1}+k_{\mathrm{p}}\right)} k_{\mathrm{z}}, \\
\theta_{\mathrm{p}}^{\prime}=\frac{k_{\mathrm{a} 1}}{k_{\mathrm{a} 1}+k_{\mathrm{p}}} \theta_{\mathrm{p}} .
\end{gathered}
$$

The equivalent mechanical models are transformed into Fig. 14. Here $C_{\mathrm{p}}^{\prime}$ is given as

$$
C_{\mathrm{p}}^{\prime}=\frac{k_{\mathrm{a} 1}+k_{\mathrm{p}}+k_{\mathrm{z}}}{k_{\mathrm{a} 1}+k_{\mathrm{p}}} C_{\mathrm{p}}^{S}
$$

From Eq. (86), the apparent elastic compliance is defined as follows on the assumption that length, width, and

(a)

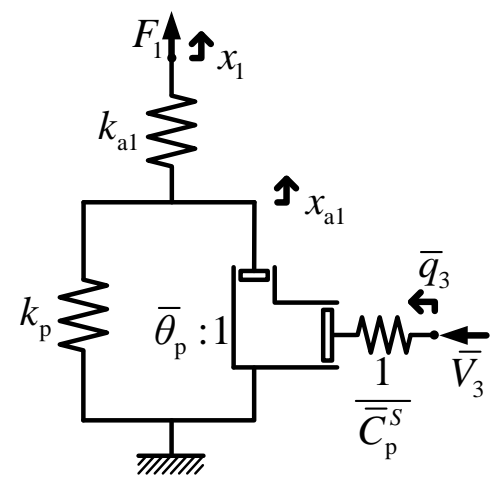

(b)

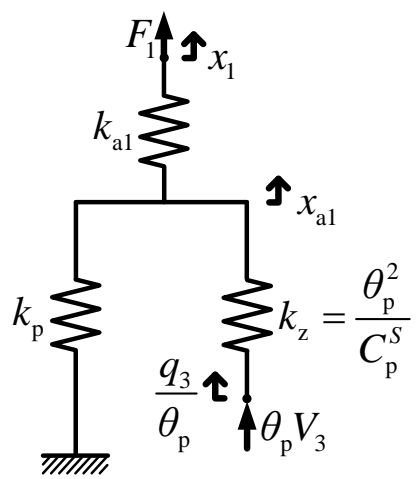

Fig. 13. Elemental equivalent mechanical models with the stiffness of the adhesive bond: (a) imaginary model and (b) perfect model. 
(a)

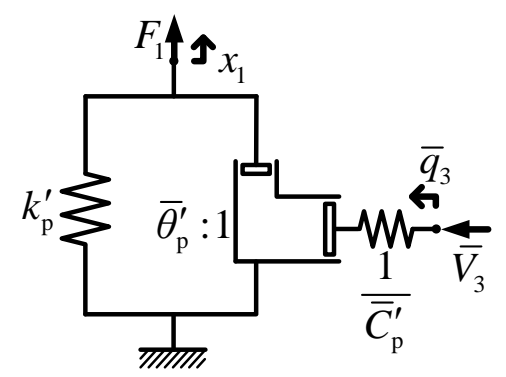

(b)

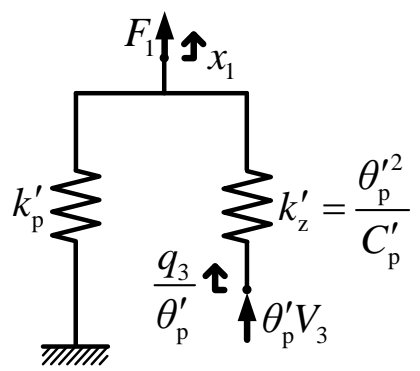

Fig. 14. Simplified elemental equivalent mechanical models with the stiffness of the adhesive bond: (a) imaginary model and (b) perfect model.

thickness of the piezoelectric element are constant.

$$
s_{11}^{\prime E}=\frac{k_{\mathrm{a} 1}+k_{\mathrm{p}}}{k_{\mathrm{a} 1}} s_{11}^{E},
$$

Apparently the value of the elastic compliance is varied by the stiffness of the adhesive bond; however, the values of the piezoelectric constant $d_{31}$ and the electrical permittivity $\varepsilon_{33}^{T}$ are not varied at all. It is difficult to predict the value of $k_{\mathrm{a} 1}$ accurately because the value depends on not only the longitudinal shear strength of the adhesive bond but also the amount and the distribution of the adhesive bond. From Eq. (90), the apparent Young's modulus of the piezoelectric element is decreased, and the decrease causes performance deterioration. Therefore, the longitudinal shear strength of the adhesive bond should be high.

\subsection{Effect of restraint in width direction}

In the preceding subsections, the properties of the piezoelectric element in the width direction are ignored for simplicity. If the Poisson's ratio of the piezoelectric element is very small and the piezoelectric element is not bound in width direction, there is no issue. However, the Poisson's ratio is generally not so small, and piezoelectric elements are usually restrained not only in the longitudinal direction but also in the width direction by the adhesive bond. To formulate the properties of the attached piezoelectric element accurately, the restraint in width direction must be taken into consideration. As shown in Fig. 15 (a), the restraint of the piezoelectric element in width direction can be expressed by the stiffness of the adhesive bond $k_{\mathrm{a} 2}$ as well as in longitudinal direction. Since the effect of the restraint in longitudinal direction has already been studied in the preceding subsection, only the effect of the restraint in width direction should be investigated in this subsection. The restraint model (in width direction only) is shown in Fig. 15 (b). The piezoelectric constitutive equations are given as,

$$
\begin{gathered}
S_{1}=s_{11}^{E} T_{1}-v_{\mathrm{p}} s_{11}^{E} T_{2}+d_{31} E_{3}, \\
0=\frac{T_{2} l_{\mathrm{p}} t_{\mathrm{p}}}{k_{\mathrm{a} 2}}+w_{\mathrm{p}}\left(-v_{\mathrm{p}} s_{11}^{E} T_{1}+s_{11}^{E} T_{2}+d_{31} E_{3}\right), \\
D_{3}=d_{31} T_{1}+d_{31} T_{2}+\varepsilon_{33}^{T} E_{3},
\end{gathered}
$$

where $v_{\mathrm{p}}$ is Poisson's ratio. The piezoelectric element was assumed to be isotropic in longitudinal and width 
(a)

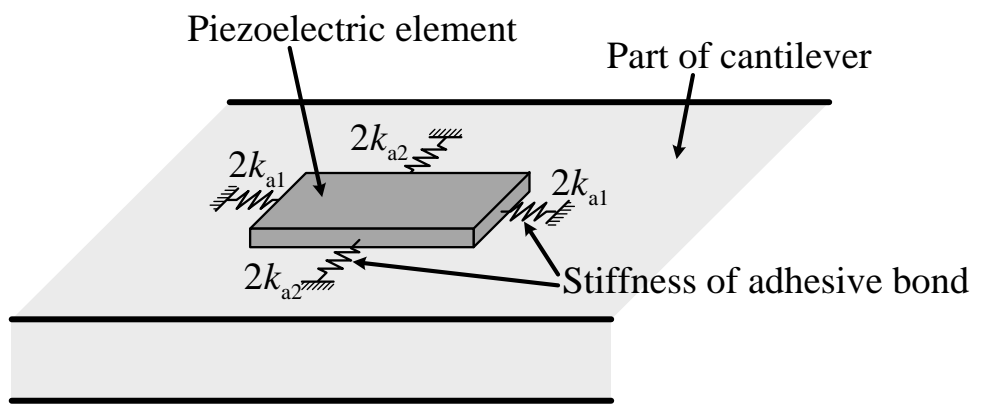

(b)

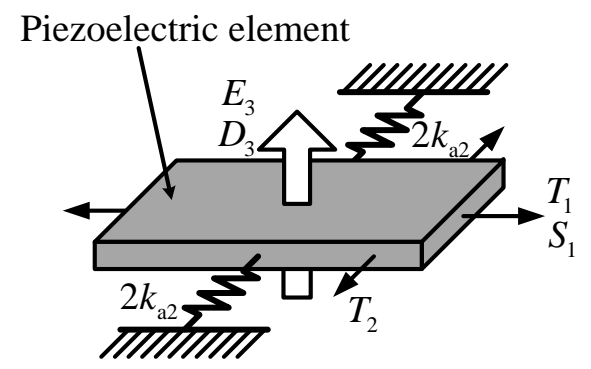

Fig. 15. Theoretical models of a piezoelectric element restrained by stiffness of the adhesive bond: (a) full restraint model and (b) restraint model only in width direction.

directions. The subscript 2 denotes the width direction. Equations (91)-(93) are transformed as follows.

$$
\begin{aligned}
& S_{1}=s_{11}^{\prime \prime E} T_{1}+d_{31}^{\prime \prime} E_{3}, \\
& D_{3}=d_{31}^{\prime \prime} T_{1}+\varepsilon_{33}^{\prime \prime T} E_{3}
\end{aligned}
$$

where

$$
\begin{gathered}
s_{11}^{\prime \prime E}=s_{11}^{E}-\frac{\left(v_{\mathrm{p}} s_{11}^{E}\right)^{2}}{s_{11}^{E}+s_{\mathrm{a} 2}}, \\
d_{31}^{\prime \prime}=d_{31}\left(1+\frac{v_{\mathrm{p}} s_{11}^{E}}{s_{11}^{E}+s_{\mathrm{a} 2}}\right), \\
\varepsilon_{33}^{\prime \prime T}=\varepsilon_{33}^{T}-\frac{d_{31}^{2}}{s_{11}^{E}+s_{\mathrm{a} 2}}, \\
s_{\mathrm{a} 2}=\frac{1}{k_{\mathrm{a} 2}} \frac{l_{\mathrm{p}} t_{\mathrm{p}}}{w_{\mathrm{p}}} .
\end{gathered}
$$

From Eqs. (96)-(98), the elastic compliance, the piezoelectric constant, and the electrical permittivity in Eqs. (1) and (2) are turned into $s_{11}^{\prime \prime E}, d_{31}^{\prime \prime}$, and $\varepsilon_{33}^{\prime \prime T}$, apparently because of the restraint in width direction. Usually the changes are larger, and all of these changes improve the performance of the piezoelectric elements. It is also difficult to obtain the accurate value of $k_{\mathrm{a} 2}$ theoretically; however, it can theoretically be said that piezoelectric elements should also be fixed in the width direction.

\section{Validation of theoretical analysis by simulation and experiment}

\subsection{Validation of optimum value of resistance}

To validate the effectiveness of the new formulations of the resistance, simulations were carried out. The simulated magnitudes of the nondimensional compliance using series and parallel LR circuits with $\beta=0.01000$ are shown in Fig. 16. Here $\zeta_{\text {opt }}$ denotes the optimum resistance ratio derived in this paper, and $\zeta_{\text {old }}$ denotes the resistance ratio adopted as an approximate optimum resistance ratio in previous papers [3-6]. The values of the resistance ratios are shown in Table 1. It is shown that the optimum resistance ratios formulated in this paper 
are superior to previous ones.

In this paper, the optimum resistance ratios were defined by root mean square of $\zeta_{\mathrm{A}}$ and $\zeta_{\mathrm{B}}$. The values of the resistance ratios derived by arithmetic average, geometric average, and root mean square are written in Table 2. It is shown that the results of three kinds of averages are almost equal, and these differences do not have influence on the frequency response functions.
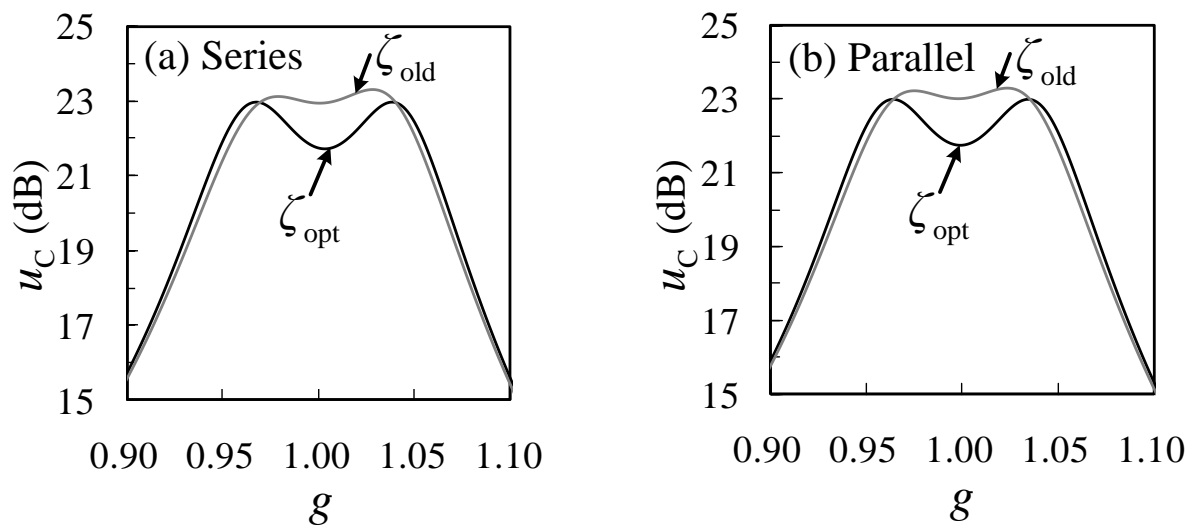

Fig. 16. Simulated magnitudes of the nondimensional compliance with two kinds of the values of the resistance and $\beta=0.01000$ : (a) series LR circuit and (b) parallel LR circuit.

\section{Table 1}

The resistance ratios used in the simulations shown in Fig. 16.

\begin{tabular}{ccc}
\hline & $\zeta_{\text {old }}$ & $\zeta_{\text {opt }}$ \\
\hline Series LR circuit & 0.07036 & 0.06108 \\
\hline Parallel LR circuit & 0.07089 & 0.06139 \\
\hline
\end{tabular}

Table 2

The resistance ratios derived by arithmetic average, geometric average, and root mean square.

\begin{tabular}{cccccc}
\hline & Point A & Point B & Arithmetic average & Geometric average & Root mean square \\
\hline Series LR circuit & 0.06320 & 0.05890 & 0.06105 & 0.06101 & 0.06108 \\
Parallel LR circuit & 0.06211 & 0.06066 & 0.06139 & 0.06138 & 0.06139 \\
\hline
\end{tabular}

\subsection{Validation of optimum values in mobility and accelerance}

\subsubsection{Experimental apparatus}

A schematic diagram of the experimental apparatus used in this study is shown in Fig. 17. The material properties of the apparatus are written in Tables 3 and 4 . Here $m_{\text {acc }}$ is the mass of the accelerometer, and $x_{\text {acc }}$ 
is the location of the accelerometer. Two pieces of piezoelectric elements were attached to the cantilever; one was used for vibration suppression and the other for excitation of the beam. Both conductive and non-conductive adhesive bonds were used. The conductive type was used to simplify wiring, and the non-conductive type for fixing and electrical insulation. Specifically, a drop of the conductive adhesive bond was placed on the center of the piezoelectric elements and it was surrounded by the non-conductive adhesive bond. An inductor made by a generalized impedance converter was used in this experiment because the size of an actual coil is too large. In this experiment, the fundamental vibration mode of the cantilever was suppressed. The equivalent stiffness ratio, the capacitance of the piezoelectric element, the modal stiffness, and the modal damping coefficient, which were experimentally measured, are shown in Table 5 [14]. Here, the superscript $\mathrm{x}$ denotes that the values were obtained experimentally. The modal stiffness were derived as

$$
K^{\mathrm{x}}=\left(2 \pi F_{\mathrm{S}}^{\mathrm{x}}\right)^{2},
$$

where $F_{\mathrm{S}}^{\mathrm{x}}$ is the natural frequency of the fundamental vibration mode when the electrodes of the piezoelectric element are shorted in the experiment. The electromechanical coupling coefficient derived by Eq. (37) and the modal mass are also written in Table 5. The experimental result of the magnitude of the nondimensional compliance with short circuit is shown in Fig. 18.

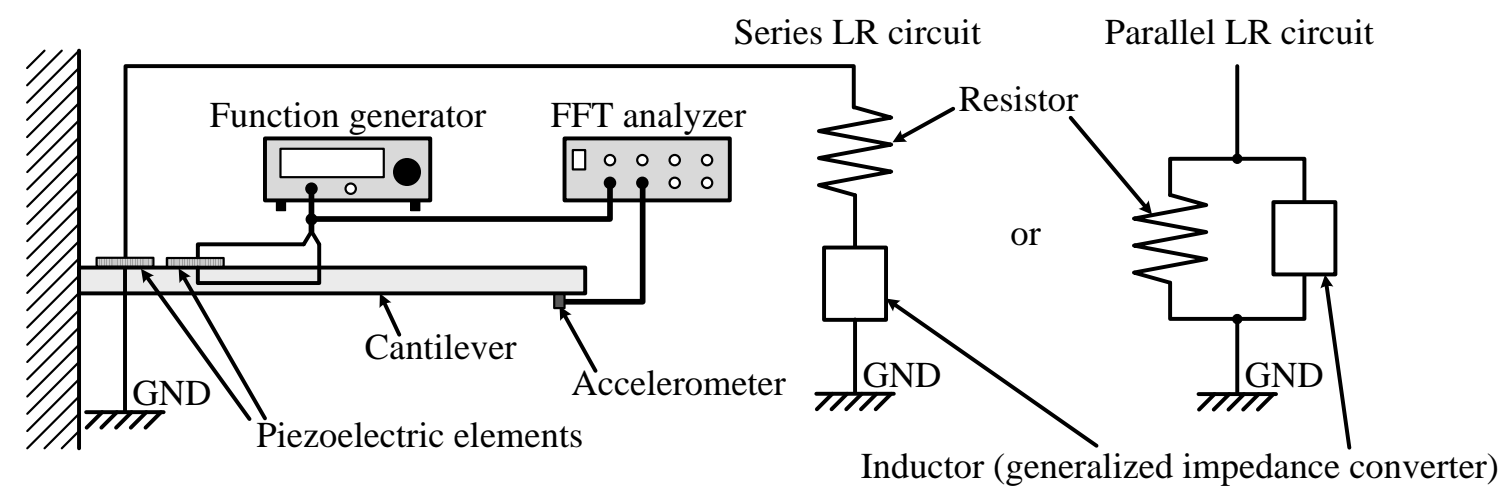

Fig. 17. Schematic diagram of the experimental apparatus.

\section{Table 3}

Material properties of the cantilever and the accelerometer.

\begin{tabular}{clcrl}
\hline \multirow{5}{*}{ Cantilever } & Length & $l_{\mathrm{b}}$ & 0.280 & $\mathrm{~m}$ \\
& Width & $w_{\mathrm{b}}$ & 0.0500 & $\mathrm{~m}$ \\
& Thickness & $t_{\mathrm{b}}$ & 0.00300 & $\mathrm{~m}$ \\
& Density & $\rho_{\mathrm{b}}$ & 7900 & $\mathrm{~kg} / \mathrm{m}^{3}$ \\
& Young's modulus & $E_{\mathrm{b}}$ & $2.06 \times 10^{11}$ & $\mathrm{~N} / \mathrm{m}^{2}$ \\
\hline \multirow{2}{*}{ Accelerometer } & Mass & $m_{\text {acc }}$ & 0.20 & $\mathrm{~g}$ \\
& Location & $x_{\text {acc }}$ & 0.270 & $\mathrm{~m}$ \\
\hline
\end{tabular}


Table 4

Material properties of the piezoelectric elements.

\begin{tabular}{lcrl}
\hline Length & $l_{\mathrm{p}}$ & 0.0320 & $\mathrm{~m}$ \\
Width & $w_{\mathrm{p}}$ & 0.0220 & $\mathrm{~m}$ \\
Thickness & $t_{\mathrm{p}}$ & 0.000230 & $\mathrm{~m}$ \\
Density & $\rho_{\mathrm{p}}$ & 8050 & $\mathrm{~kg} / \mathrm{m}^{3}$ \\
Elastic compliance & $s_{11}^{E}$ & $1.55 \times 10^{-11}$ & $\mathrm{~m}^{2} / \mathrm{N}$ \\
Young's modulus & $E_{\mathrm{p}}$ & $6.45 \times 10^{10}$ & $\mathrm{~N} / \mathrm{m}^{2}$ \\
Piezoelectric constant & $d_{31}$ & $2.30 \times 10^{-10}$ & $\mathrm{C} / \mathrm{N}$ \\
Electrical permittivity & $\varepsilon_{33}^{T}$ & $2.35 \times 10^{-8}$ & $\mathrm{~F} / \mathrm{m}$ \\
Poisson's ratio & $v_{\mathrm{p}}$ & 0.30 & \\
Dielectric loss factor & $\delta$ & 0.020 & \\
Location of the piezoelectric element & $\left(x_{1}, x_{\mathrm{r}}\right)$ & $(0.0050,0.0370)$ & $\mathrm{m}$ \\
for vibration suppression & & & \\
Location of the piezoelectric element & $\left(x_{1}, x_{\mathrm{r}}\right)$ & $(0.0400,0.0720)$ & $\mathrm{m}$ \\
for excitation & & & \\
\hline
\end{tabular}

Table 5

Experimentally measured parameters of the cantilever with the two piezoelectric elements.

\begin{tabular}{llrl}
\hline Equivalent stiffness ratio & $\beta^{\mathrm{x}}$ & 0.00411 & \\
Capacitance & $C_{\mathrm{p}}^{\mathrm{x}}$ & 0.0480 & $\mu \mathrm{F}$ \\
Electromechanical coupling coefficient & $\Theta^{\mathrm{x}}$ & 0.00273 & $\mathrm{~N} / \mathrm{V}$ \\
Modal stiffness & $K^{\mathrm{x}}$ & 37800 & $\mathrm{~N} / \mathrm{m}$ \\
Modal damping coefficient & $D^{\mathrm{x}}$ & 0.567 & $\mathrm{Ns} / \mathrm{m}$ \\
Modal mass & $M$ & 1.00 & $\mathrm{~kg}$ \\
\hline
\end{tabular}

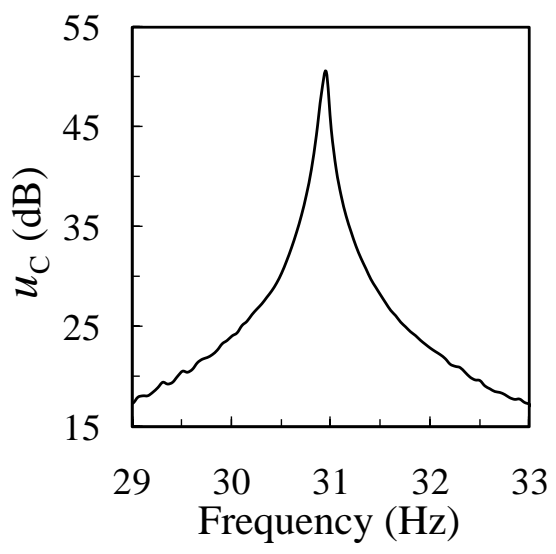

Fig. 18. The experimental result of the magnitude of the nondimensional compliance with short circuit. 


\subsubsection{Simulated and experimental results}

The simulated and experimental magnitudes of the nondimensional compliance, mobility, and accelerance using optimum values in compliance, mobility, and accelerance, respectively, are shown in Figs. 19 and 20,
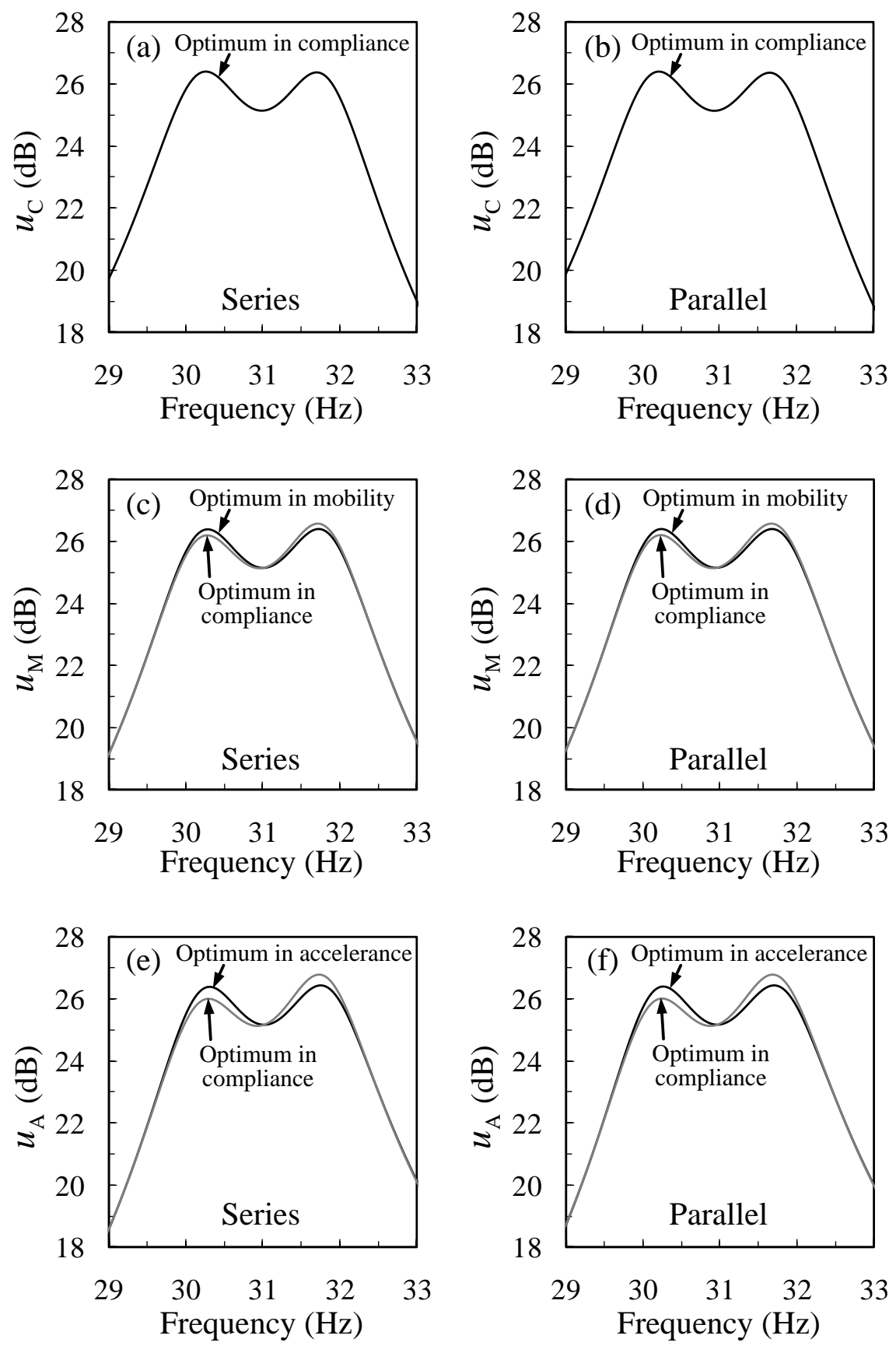

Fig. 19. Simulated results of magnitudes of the nondimensional compliance, mobility, and accelerance using the optimum values: (a) compliance with series LR circuit, (b) compliance with parallel LR circuit, (c) mobility with series LR circuit, (d) mobility with parallel LR circuit, (e) accelerance with series LR circuit, and (f) accelerance with parallel LR circuit. 

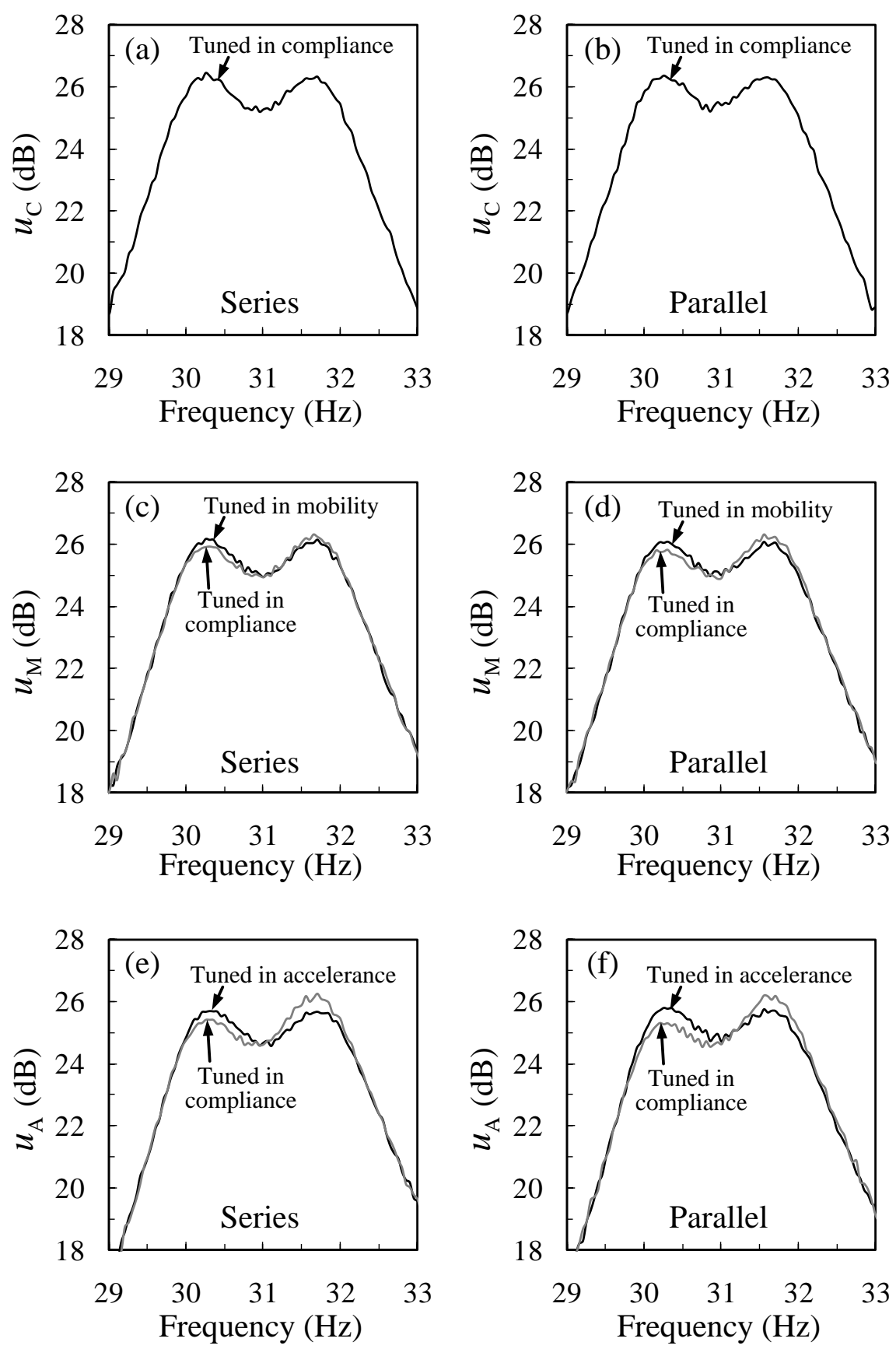

Fig. 20. Experimental results of magnitudes of the nondimensional compliance, mobility, and accelerance using the optimum values: (a) compliance with series LR circuit, (b) compliance with parallel LR circuit, (c) mobility with series LR circuit, (d) mobility with parallel LR circuit, (e) accelerance with series LR circuit, and (f) accelerance with parallel LR circuit.

respectively. The values of the material properties used in the simulations are the experimental ones written in Table 5. The damping coefficient of the cantilever was ignored in the theoretical analysis; however, it was included in these simulations. In the mobility and the accelerance, the results using the LR circuit which was 
tuned optimally in terms of the compliance are also shown. The theoretical optimum values and the experimental values of the LR circuit are listed in Tables 6 and 7, respectively. These frequency response functions change sensitively depending on the value of the inductance. The magnitude relation of the inductance values in the experiment agrees well with the simulated ones. The reason why the values of the inductance are large is that the capacitance value of the piezoelectric element is small. The theoretical optimum values and the experimental ones of the resistance are much different. The detail is described in the following subsection. It can be said that optimum values in the proper frequency response function should be adopted.

Table 6

Theoretical optimum values of the LR circuit in compliance, mobility, and accelerance.

\begin{tabular}{cccccc}
\hline & & Compliance & Mobility & Accelerance & \\
\hline \multirow{2}{*}{ Series LR circuit } & $L_{\mathrm{opt}}$ & 549 & 548 & 547 & $\mathrm{H}$ \\
\cline { 2 - 6 } & $R_{\mathrm{opt}}$ & $8.39 \times 10^{3}$ & $8.37 \times 10^{3}$ & $8.36 \times 10^{3}$ & $\Omega$ \\
\hline \multirow{2}{*}{ Parallel LR circuit } & $L_{\mathrm{opt}}$ & 552 & 551 & 550 & $\mathrm{H}$ \\
\cline { 2 - 6 } & $R_{\mathrm{opt}}$ & $1.36 \times 10^{6}$ & $1.36 \times 10^{6}$ & $1.36 \times 10^{6}$ & $\Omega$ \\
\hline
\end{tabular}

Table 7

Experimental values of the LR circuit in compliance, mobility, and accelerance.

\begin{tabular}{cccccc}
\hline & & Compliance & Mobility & Accelerance & \\
\hline \multirow{2}{*}{ Series LR circuit } & $L$ & 553 & 552 & 550 & $\mathrm{H}$ \\
\cline { 2 - 6 } & $R$ & $5.10 \times 10^{3}$ & $5.10 \times 10^{3}$ & $5.10 \times 10^{3}$ & $\Omega$ \\
\hline \multirow{2}{*}{ Parallel LR circuit } & $L$ & 558 & 555 & 555 & $\mathrm{H}$ \\
\cline { 2 - 6 } & $R$ & $2.41 \times 10^{6}$ & $2.41 \times 10^{6}$ & $2.41 \times 10^{6}$ & $\Omega$ \\
\hline
\end{tabular}

\subsection{Various resistance ratios in experiment}

The difference between the theoretical and experimental resistance values in Tables 6 and 7 is due to the dielectric loss of the piezoelectric element and the internal resistance of the inductor made using the generalized impedance converter. $R_{C}$ and $R_{L}$ measured experimentally are written in Table 8. Here $R_{C}$ was measured at $\omega=\left(K^{\mathrm{x}}\right)^{1 / 2} \cdot R_{C}$ obtained by Eq. (72) and $\omega=\left(K^{\mathrm{x}}\right)^{1 / 2}$ is also written in Table 8 in parentheses. The various resistance ratios in the experiment and the theoretical optimum resistance ratios are given in Table 8. Only the values with respect to compliance are listed because the difference among compliance, mobility, and accelerance is small. The experimental total resistance ratios and the theoretical optimum resistance ratios agree well. These results imply that the optimum resistance value in the experiment can be estimated and dielectric loss of piezoelectric elements should be taken into consideration when the material of the piezoelectric elements is determined. 
Table 8

Values of $R_{C}, R_{L}$, and various resistance ratios in the experiment and theoretical optimum resistance ratios.

\begin{tabular}{|c|c|c|c|c|}
\hline & & Series LR & Parallel LR & \\
\hline \multirow{7}{*}{ Experimental values } & $R_{C}$ & \multicolumn{2}{|c|}{$3.61 \times 10^{6}\left(3.6 \times 10^{6}\right)$} & $\Omega$ \\
\hline & $R_{L}$ & \multicolumn{2}{|c|}{37} & $\Omega$ \\
\hline & $\zeta_{C}$ & 0.0149 & 0.0149 & \\
\hline & $\zeta_{L}$ & 0.00017 & 0.00017 & \\
\hline & $\zeta_{\mathrm{s}}$ & 0.0238 & - & \\
\hline & $\zeta_{\mathrm{P}}$ & - & 0.0224 & \\
\hline & $\zeta_{\mathrm{T}}$ & 0.0389 & 0.0375 & \\
\hline Theoretical optimum values & $\zeta_{\mathrm{opt}}$ & 0.0392 & 0.0393 & \\
\hline
\end{tabular}

\subsection{Investigation of stiffness of adhesive bond in experiment}

To investigate the effect of the adhesive bond, a calculation model was constructed as shown in Fig. 21. The accurate curvature of the mode shape of the cantilever can be derived by the calculation model. The cantilever was divided into 280 segments, and the equivalent mass and stiffness of each segment were theoretically obtained. The stiffness and the mass of the two piezoelectric elements and the mass of the accelerometer were taken into consideration. The theoretically calculated equivalent stiffness ratio $\beta^{\mathrm{c}}$, capacitance $C_{\mathrm{p}}^{\mathrm{c}}$, modal stiffness $K^{\mathrm{c}}$, and modal electromechanical coupling coefficient $\Theta^{\mathrm{c}}$ using various $k_{\mathrm{a} 1}$ and $k_{\mathrm{a} 2}$ are shown in Fig. 22. The material parameters listed in Tables 3 and 4 were used in this calculation. Note that the axes of $C_{\mathrm{p}}^{\mathrm{c}}$ are reversed. The results show that the equivalent stiffness ratio and the capacitance of the piezoelectric element are greatly affected by the stiffness of the adhesive bond. Those values are varied significantly around $k_{\mathrm{a} 1}=k_{\mathrm{p}}=1.02 \times 10^{7} \mathrm{~N} / \mathrm{m}$ and $k_{\mathrm{a} 2}=k_{\mathrm{p} 2}=2.16 \times 10^{7} \mathrm{~N} / \mathrm{m}$. Here

$$
k_{\mathrm{p} 2}=E_{\mathrm{p}} \frac{l_{\mathrm{p}} t_{\mathrm{p}}}{w_{\mathrm{p}}} .
$$

The theoretically calculated combinations of $k_{\mathrm{a} 1}$ and $k_{\mathrm{a} 2}$, which satisfy either $\beta^{\mathrm{c}}=0.00411$ or $C_{\mathrm{p}}^{\mathrm{c}}=0.0480 \mu \mathrm{F}$, are shown in Fig. 23. The values of the intersection point are $k_{\mathrm{a} 1}=1.26 \times 10^{8} \mathrm{~N} / \mathrm{m}$ and $k_{\mathrm{a} 2}=7.76 \times 10^{7} \mathrm{~N} / \mathrm{m}$. These are the estimated values of $k_{\mathrm{a} 1}$ and $k_{\mathrm{a} 2}$ in the experimental apparatus. Both $k_{\mathrm{a} 1}$ and $k_{\mathrm{a} 2}$ are larger than $k_{\mathrm{p}}$ and $k_{\mathrm{p} 2}$, respectively; however, the value of the equivalent stiffness ratio can still be improved by using stiffer adhesive bonds.

The reason why $k_{\mathrm{a} 2}$ is smaller than $k_{\mathrm{a} 1}$ is that the distribution of the adhesive bond were not uniform in the experimental apparatus. An extreme example is shown in Fig. 24. In this example, the piezoelectric element is restrained only in the longitudinal direction. By comparison, $k_{\mathrm{a} 1}$ and $k_{\mathrm{a} 2}$ become the same value if the adhesive bond is uniformly spread on the entire piezoelectric element. Since the difference between the estimated values of $k_{\mathrm{a} 1}$ and $k_{\mathrm{a} 2}$ is not so large, it is reasonable to suppose that the theoretical analysis with 
respect to adhesive bonds is effective.

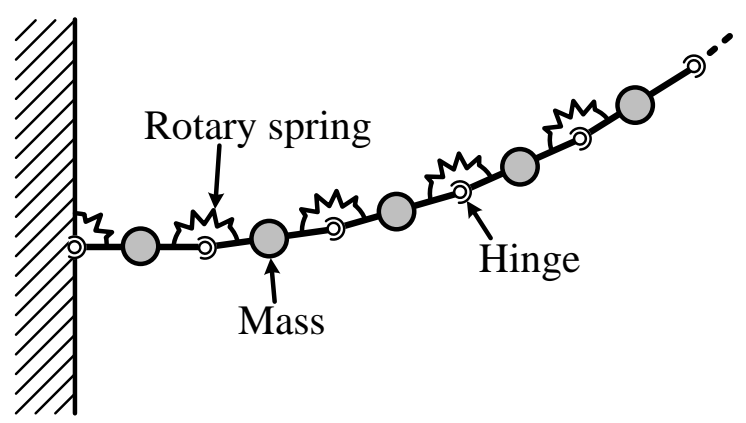

Fig. 21. Calculation model of the cantilever with the piezoelectric elements to derive the mode shape.
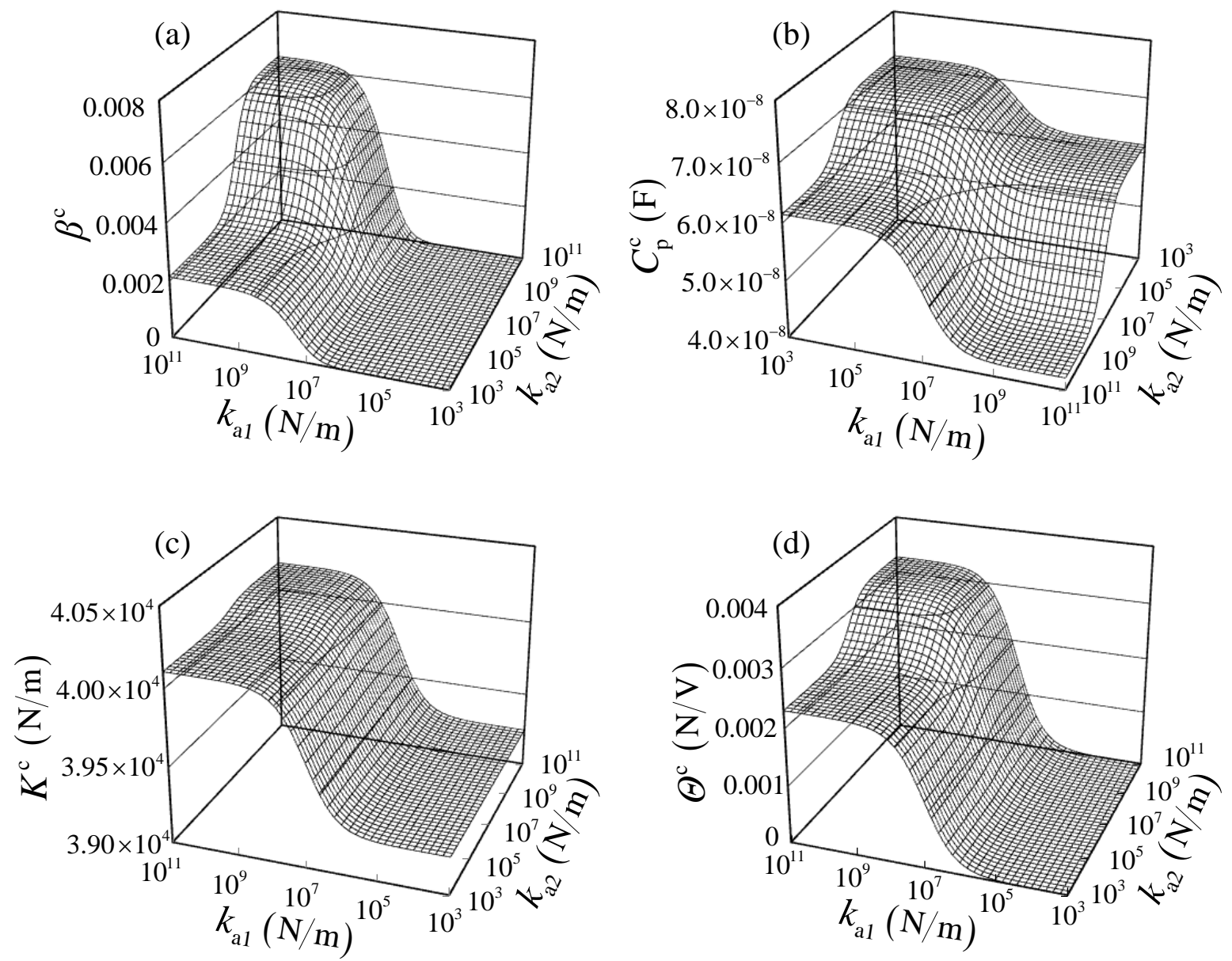

Fig. 22. Theoretically calculated parameters of the cantilever with the piezoelectric elements using various $k_{\mathrm{a} 1}$ and $k_{\mathrm{a} 2}$ : (a) equivalent stiffness ratio, (b) capacitance, (c) modal stiffness, and (d) electromechanical coupling coefficient. 


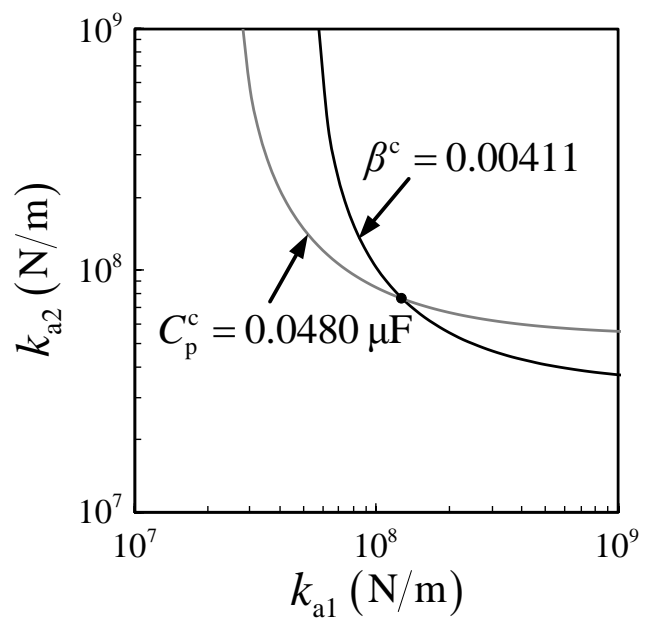

Fig. 23. Theoretically calculated results of the combinations of $k_{\mathrm{a} 1}$ and $k_{\mathrm{a} 2}$ which satisfy $\beta^{\mathrm{c}}=0.00411$ and $C_{\mathrm{p}}^{\mathrm{c}}=0.0480 \mu \mathrm{F}$, respectively.

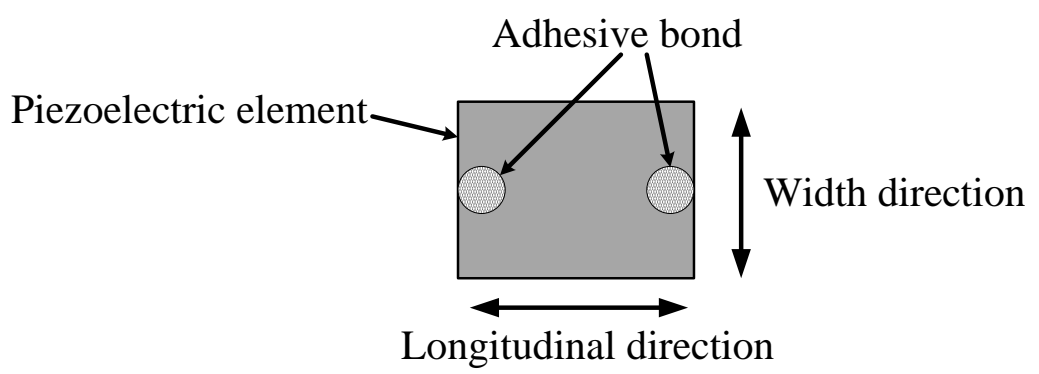

Fig. 24. An extreme example of the distribution of the adhesive bond.

\section{Conclusion}

The governing equations for passive vibration suppression with series and parallel LR circuits were derived using the new equivalent mechanical model of a piezoelectric element. The optimum values of the series and the parallel LR circuits were formulated by using the two fixed points method not only in terms of compliance but also in terms of mobility and accelerance. The difference between the series and parallel LR circuits was investigated theoretically. The effects of the dielectric loss of the piezoelectric elements, the internal resistance of the inductor, and the stiffness of adhesive bonds were theoretically investigated. The theoretical analysis was validated through numerical simulations and experiments. The performance of the passive vibration suppression technique using LR circuits was determined based on the value of the equivalent stiffness ratio as well as the mass ratio in typical mechanical vibration absorbers. The series LR circuit is superior to the parallel one in terms of compliance and mobility. However, the parallel LR circuit is superior to the series one in terms of robustness with respect to the variation of the resistance. Therefore, it is necessary to use two kinds of LR circuits properly according to the host structure and the piezoelectric element. This work demonstrated that the total resistance ratio, which includes the dielectric loss of the piezoelectric element and the internal resistance of the inductor, 
should be tuned to be equal to the optimum resistance ratio, and an adhesive bond with high longitudinal shear strength should be used.

\section{Acknowledgement}

This study was partly supported by Grant-in-Aid for Young Scientists (B) (JSPS, No. 21760169).

\section{References}

[1] R. L. Forward, Electronic damping of vibrations in optical structures, Applied Optics 18 (5) (1979) 690-697.

[2] J. J. Dosch, D. J. Inman and E. Garcia, A self-Sensing piezoelectric actuator for collocated control, Journal of Intelligent Material Systems and Structures 3 (1992) 166-185.

[3] N. W. Hagood and A. Von Flotow, Damping of structural vibrations with piezoelectric materials and passive electrical networks, Journal of Sound and Vibration 146 (2) (1991) 243-268.

[4] C. H. Park, Dynamics modeling of beams with shunted piezoelectric elements, Journal of Sound and Vibration 268 (1) (2003) 115-129.

[5] S. Y. Wu, Piezoelectric shunts with a parallel R-L circuit for structural damping and vibration control, Proceedings of the SPIE 2720, San Diego, February 1996, 259-269.

[6] C. H. Park and D. J. Inman, A uniform model for series R-L and parallel R-L shunt circuits and power consumption, Proceedings of the SPIE 3668, California, March 1999, 797-804.

[7] K. Yamada, H. Matsuhisa and H. Utsuno, Passive vibration suppression using two inductances and piezoelectric elements, Proceedings of the 12th Asia Pacific Vibration Conference, Sapporo, August 2007, CD-ROM 114.

[8] G. S. Agnes, Development of a modal model for simultaneous active and passive piezoelectric vibration suppression, Journal of Intelligent Material Systems and Structures 6 (1995) 482-487.

[9] M. S. Tsai and K. W. Wang, On the structural damping characteristics of active piezoelectric actuators with passive shunt, Journal of Sound and Vibration 221 (1) (1999) 1-22.

[10] K. Adachi, Y. Awakura and T. Iwatsubo, Hybrid piezoelectric damping for structural vibration suppression, Journal of Intelligent Material Systems and Structures 15 (2004) 795-801.

[11] K. Yamada, H. Matsuhisa , H. Utsuno and J. G. Park, Hybrid vibration suppression of flexible structures using piezoelectric elements and analog circuits, JSME Journal of Environment and Engineering 3 (2) (2008) 424-435.

[12] J. Ormondroyd and J. P. Den Hartog, The theory of the dynamic vibration absorber, Transactions of the American Society of Mechanical Engineers 50 (7) (1928) 9-22.

[13] H. H. Law, P. L. Rossiter, G. P. Simon, and L. L. Koss, Characterization of mechanical vibration damping by piezoelectric materials, Journal of Sound and Vibration 197 (4) (1996) 489-513.

[14] K. Yamada, H. Matsuhisa , H. Utsuno and J. G. Park, Precise measurement technique of the 
electromechanical coupling coefficient of piezoelectric elements, Proceedings of the 8th International Conference on Motion and Vibration Control, Daejeon, August 2006, Flash memory MA1-3. 